\title{
Fabrication, Performance, and Model for Proton Conductive Solid Oxide Fuel Cell
}

\author{
Chih-Long Tsai*,z and V. Hugo Schmidt**
}

Department of Physics, Montana State University, Bozeman, Montana 59717

$\mathrm{Ba}\left(\mathrm{Ce}_{0.8} \mathrm{Y}_{0.2}\right) \mathrm{O}_{3-\delta}$ anode-supported proton conductive solid oxide fuel cells (H-SOFC) were fabricated and tested under different $\mathrm{H}_{2}$ and $\mathrm{O}_{2}$ partial pressures and temperatures. An electrochemical model developed from Boltzmann statistics was derived to study the $V(i)$ of the H-SOFC and proton conductive solid oxide electrolyzer (H-SOEC). Our model has successfully fit the $V(i)$ curves to our variety of $\mathrm{H}_{2} / \mathrm{O}_{2}$ partial pressure expermental data with only one adjustable parameter. By using the model, the parametric analyses suggest the major possible and easiest way to improve the already good performance of the $\mathrm{H}$-SOFC is reducing its electrolyte thickness. Other than that, the performance of H-SOFC is mostly restricted by the attempt current density $c$, which means the cathode of the H-SOFC limits the performance of the whole cell. The H-SOFC performance can be enhanced by carefully engineering its cathode to increase its triple phase boundaries or finding a cathode material that enables more $\mathrm{O}_{2}$ molecules to be adsorbed and dissociate into available $\mathrm{O}$ atoms on the cathode surface.

(C) 2011 The Electrochemical Society. [DOI: 10.1149/1.3596361] All rights reserved.

Manuscript submitted November 18, 2010; revised manuscript received May 9, 2011. Published June 7, 2011.

Solid oxide fuel cells (SOFCs) provide clean and efficient generation of electricity using a variety of fuels. Present SOFCs operate in the $800-1000^{\circ} \mathrm{C}$ temperature range, however, strong efforts are being made to lower the operating temperature down to the 800$600^{\circ} \mathrm{C}$ range. By lowering the operating temperature down to near $600^{\circ} \mathrm{C}$, stainless steel can be used for the interconnect to minimize system cost and also reduce problems such as thermal expansion mismatch, atomic migration, and corrosion. The lower activation energy for proton conductivity compared to oxygen ion conductivity, allows proton conductive solid oxide fuel cells (H-SOFCs) to be operated at a relative low temperature. Furthermore, total fuel utilization without a complicated hydrogen recirculation system is plausible for the H-SOFCs, which helps make them more attractive than the conventional oxygen ion conductive solid oxide fuel cells (O-SOFCs).

Presently, a lot of experimental work but not much electrochemical modeling has been done on the H-SOFCs. In this paper, we use molecular dynamics to model the $i-V$ (current density vs. voltage) curve instead of using the Nernst and Butler-Volmer equations. The analysis of the $i$ - $V$ curve for the H-SOFCs is based on the concept of exchange current density, $i_{0}$. The exchange current density is defined for open-circuit operation in which equal and opposite current densities in the system result from the equal forward and reverse reaction rates at the anode-electrolyte and cathode-electrolyte interfaces. Accordingly, there are two exchange current densities, $i_{0 a}$ from the reaction at the triple phase boundary (TPB) where the anode, anode pores, and electrolyte meet, and $i_{0 c}$ from the reaction at the TPB where the cathode, cathode pores, and electrolyte meet.

For closed-circuit operation, we continue to use the concept of forward and reverse reactions and their corresponding current densities. The net current density across the cell comes from the difference between the forward and reverse current densities, each of which is described as the product of an attempt current density and a reaction success probability.

By normalizing these reaction success probabilities, we obtain expressions for current density as a function of anode or cathode activation polarization that give us the correct saturation current density in the limit of large applied voltage in both the SOFC and SOEC mode.

Our $V(i)$ expression is found by including concentration polarization $V_{\text {conc }}(i)$, which accounts for variation with $i$ of gas concentrations at the anode-electrolyte and cathode-electrolyte interfaces. We also include ohmic polarization $V_{\text {ohm }}(i)$ by introducing proton

\footnotetext{
* Electrochemical Society Student Member

** Electrochemical Society Active Member.

z E-mail: tsai@physics.montana.edu
}

and electronic conductivity of the electrolyte, which accounts for ohmic voltage drops from transporting ions and electrons through the electrolyte. Finally, we use our H-SOFC experimental data to check the accuracy of our model.

\section{Experimental}

The $\mathrm{Ba}\left(\mathrm{Ce}_{0.8} \mathrm{Y}_{0.2}\right) \mathrm{O}_{3-\delta}$ powder was prepared by solid state reaction. Stoichiometric amounts of barium carbonate $\left(\mathrm{BaCO}_{3}, 99.8 \%\right)$, cerium oxide $\left(\mathrm{CeO}_{2}, 99.97 \%\right)$, and yttrium oxide $\left(\mathrm{Y}_{2} \mathrm{O}_{3}, 99.99 \%\right)$ from Inframat Advanced Materials were mixed by an agate autogrinding machine for two hours. Then, the powder was calcined at $1100^{\circ} \mathrm{C}$ for $15 \mathrm{~h}$ to form the perovskite phase. An X-ray diffraction (Scintag, XGEN-400) with $\mathrm{Cu} \mathrm{K} \alpha(\lambda=1.5418 \AA$ ) was used for checking the formation of $\mathrm{Ba}\left(\mathrm{Ce}_{0.8} \mathrm{Y}_{0.2}\right) \mathrm{O}_{3-\delta}$. The auto-grinding and calcining processes were repeated until a single phase of the material was confirmed by the $\mathrm{X}$-ray diffraction.

To prepare supporting anodes, the $\mathrm{Ba}\left(\mathrm{Ce}_{0.8} \mathrm{Y}_{0.2}\right) \mathrm{O}_{3-\delta}$ powder was weighed and mixed with $\mathrm{NiO}$ at volume ratio $1: 2$. In addition to ceramic powders, $10 \mathrm{wt} \%$ cornstarch of the total solid load was added to the powder and auto-grinded for $2 \mathrm{~h}$ to serve as pore former. The powder was uniaxial die-pressed with a $3 / 4$ " die to form green pellets to serve as supporting anodes. Then the anode interlayer, with a 1:1 volume ratio of $\mathrm{Ba}\left(\mathrm{Ce}_{0.8} \mathrm{Y}_{0.2}\right) \mathrm{O}_{3-\delta}$ to $\mathrm{NiO}$, and the electrolyte, $\mathrm{Ba}\left(\mathrm{Ce}_{0.8} \mathrm{Y}_{0.2}\right) \mathrm{O}_{3-\delta}$, were planetary ball milled with ethanol for $4 \mathrm{~h}$ at $150 \mathrm{rpm}$. After drying in the oven, the powders were mixed with alpha terpinol, ethylcellulose, oleic acid and xylene using a three roll miller to form inks. The inks were paint brushed on the supporting anode. After the drying of electrolyte ink, the pellets were re-pressed by the uniaxial die press. To prevent barium evaporation at elevated temperature, $\mathrm{Ba}\left(\mathrm{Ce}_{0.8} \mathrm{Y}_{0.2}\right) \mathrm{O}_{3-\delta}$ powder was used to cover the cells when sintering, about $5 \mathrm{~mm}$ thick of $\mathrm{Ba}\left(\mathrm{Ce}_{0.8} \mathrm{Y}_{0.2}\right) \mathrm{O}_{3-\delta}$ powder on the top and below the cell. The cells were then sintered in air at a temperature of $1400^{\circ}$ for $5 \mathrm{~h}$ to form a well-bonded electrolyte-anode structure. Cathode material ink, $\mathrm{La}_{0.8} \mathrm{Sr}_{0.2} \mathrm{MnO}_{3 \pm \delta}$ (LSM), was then applied on the sintered pellets by paint brush and fired to $900^{\circ} \mathrm{C}$ in air for $2 \mathrm{~h}$ to form a good bond between cathode and electrolyte.

A home-made seal-less testing system made from Inconel 600 was used for the measurements. Silver mesh and nickel foam were used as cathode and anode current collectors, respectively. Silver paste was used to adhere the current collectors to the electrodes of the tested cell. The total input gas flow rates on each side were controlled by MKS mass flow controllers at $200 \mathrm{ml} \cdot \mathrm{min}^{-1}$ in all experiments. The water vapor concentration in fuel gas was about $3 \%$ by flowing the fuel gas through a water bubbler at room temperature. Measurements were carried out at temperature between 650 and $800{ }^{\circ} \mathrm{C}$ with $50^{\circ} \mathrm{C}$ temperature steps in ambient pressure. The cells 


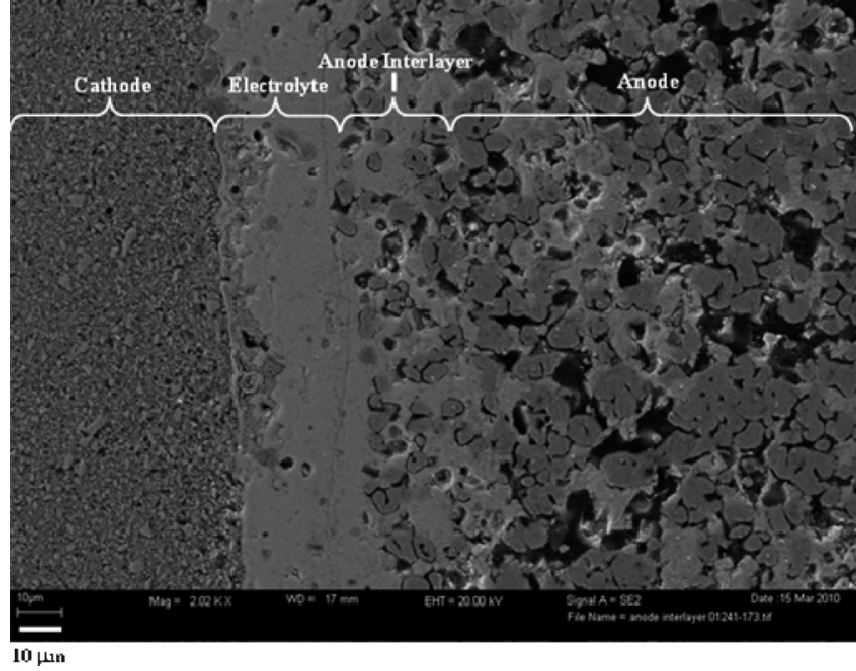

Figure 1. Microstructure of $\mathrm{Ba}\left(\mathrm{Ce}_{0.8} \mathrm{Y}_{\mathbf{0 . 2}}\right) \mathrm{O}_{3-\delta}$ anode supported H-SOFC from SEM image.

were reduced in situ at high temperature in a $60 \% \mathrm{H}_{2}+40 \% \mathrm{~N}_{2}$ mixture for more than an hour prior to the measurements. All performance of the cell was measured using various fuel gas mixtures, i.e. at various partial pressures of $\mathrm{H}_{2} / \mathrm{N}_{2}$ ratio in fuel gas while the total flow rate of the gas was kept constant at $200 \mathrm{ml} \mathrm{min}^{-1}$. The current densities were calculated based on the cathode area.

Porosity of the supporting anode was measured using Archimedes' method. The tested cell, after being cooled in a reducing atmosphere to prevent $\mathrm{NiO}$ formation, was broken into two pieces. One of the pieces was polished to erase the cathode and electrolyte layers and cleaned with an ultrasonic bath in ethanol. The sample was then kept in a dry oven at $95^{\circ} \mathrm{C}$ for more than $2 \mathrm{~h}$ to evaporate the ethanol. Dry weight, $\mathrm{W}_{\mathrm{dry}}$, wet weight, $\mathrm{W}_{\text {wet }}$, and weight saturated with ethanol, $\mathrm{W}_{\text {sat }}$, were measured using a high accuracy balance. Prior to measuring $\mathrm{W}_{\text {wet }}$ in ethanol, the sample was immersed in ethanol and kept in vacuum for $10 \mathrm{~min}$ to remove possible air in the pores. The $\mathrm{W}_{\text {sat }}$ was measured in air soon after the surface of the sample was shaken dry. The open porosity was calculated by using the equation

$$
\text { Porosity }(\%)=\frac{W_{\text {sat }}-W_{\text {dry }}}{W_{\text {sat }}-W_{\text {wet }}} \times 100 \%
$$

The other part of the cell was coated with epoxy that was allowed to harden, and then was polished. Field Emission Scanning Electron Microscopy (SUPRA $^{\mathrm{TM}} 55$ Versatile High Performance FE-SEM, Zeiss) was used to examine the microstructure of the cell. The mean pore radius of the cell was determined by quantitative measurements of the pore size on SEM images.

\section{Results}

Figure 1 shows the microstructure of the tested cell. The dense electrolyte is $\sim 30 \mu \mathrm{m}$ in thickness, to which adheres a $\sim 25 \mu \mathrm{m}$ anode interlayer. The average pore size of the supporting anode is $\sim 1.42 \mu \mathrm{m}$ in diameter, which was calculated quantitatively from SEM pictures. The thickness of the supporting anode is about 1.15 $\mathrm{mm}$ with porosity $\sim 44 \%$. The cathode thickness is $\sim 50 \mu \mathrm{m}$. The effective cathode area of the cell is $0.57 \mathrm{~cm}^{2}$.

The temperature dependent electrochemical performance of an anode supported $\mathrm{Ba}\left(\mathrm{Ce}_{0.8} \mathrm{Y}_{0.2}\right) \mathrm{O}_{3-\delta} \mathrm{H}$-SOFC using $100 \% \mathrm{H}_{2}$ as fuel gas is shown in Fig. 2. The peak power densities are 1145, 953, 763 and $544 \mathrm{~mW} / \mathrm{cm}^{2}$ at temperature $800,750,700$ and $650^{\circ} \mathrm{C}$, respectively. This performance is very good, especially at low temperature ranges when compared to oxygen ion conductor cells. For instance, a cell with $10 \mu \mathrm{m}$ thick 8 YSZ electrolyte and LSM-8YSZ cathode showed $850,625,430$ and $275 \mathrm{~mW} / \mathrm{cm}^{2}$ at temperature 800,750 , 700 and $650^{\circ}$, respectively. ${ }^{1}$ The high open circuit voltage, $\sim 1 \mathrm{~V}$ at $700^{\circ} \mathrm{C}$, indicates the $\mathrm{Ba}\left(\mathrm{Ce}_{0.8} \mathrm{Y}_{0.2}\right) \mathrm{O}_{3-\delta}$ electrolyte is sufficiently dense; however, a much lower open-circuit voltage when compared to Nernst potential suggests the electronic conduction was high at high temperature. For $\mathrm{Ba}\left(\mathrm{Ce}_{0.8} \mathrm{Y}_{0.2}\right) \mathrm{O}_{3-\delta}$ ceramic, a reducing oxygen partial pressure resulted in a transition from dominant p-type electronic to ionic conduction, followed by n-type electronic conduction. This is due to the redox reaction of $\mathrm{Ce}^{+3} / \mathrm{Ce}^{+4}$ in $\mathrm{Ba}\left(\mathrm{Ce}_{0.8} \mathrm{Y}_{0.2}\right) \mathrm{O}_{3-\delta}$ under reducing atmosphere, which increases the

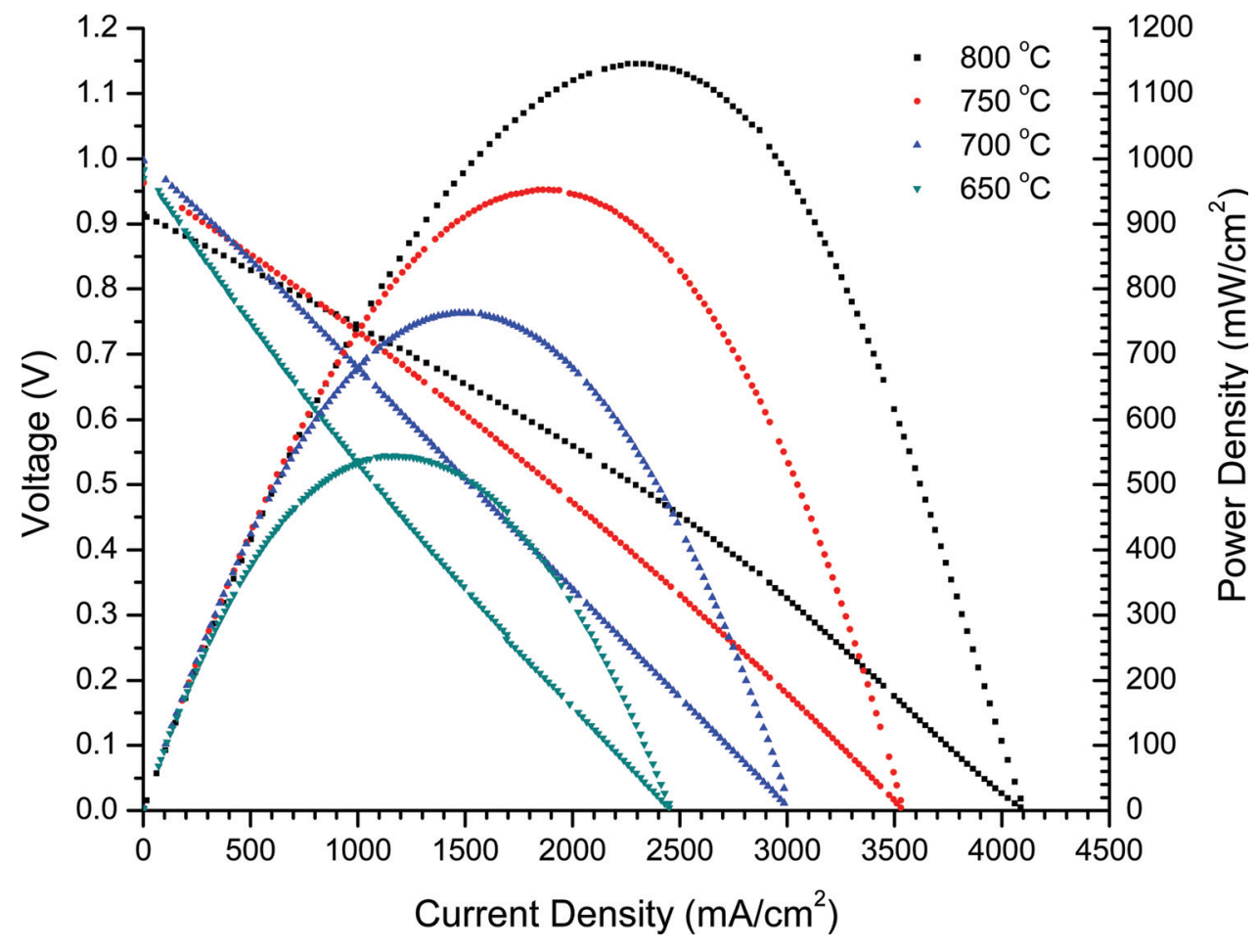

Figure 2. (Color online) Temperature dependent electrochemical performances of $\mathrm{Ba}\left(\mathrm{Ce}_{0.8} \mathrm{Y}_{\mathbf{0 . 2}}\right) \mathrm{O}_{3-\delta}$ anode supported cell using $100 \% \mathrm{H}_{2}$ as fuel. 

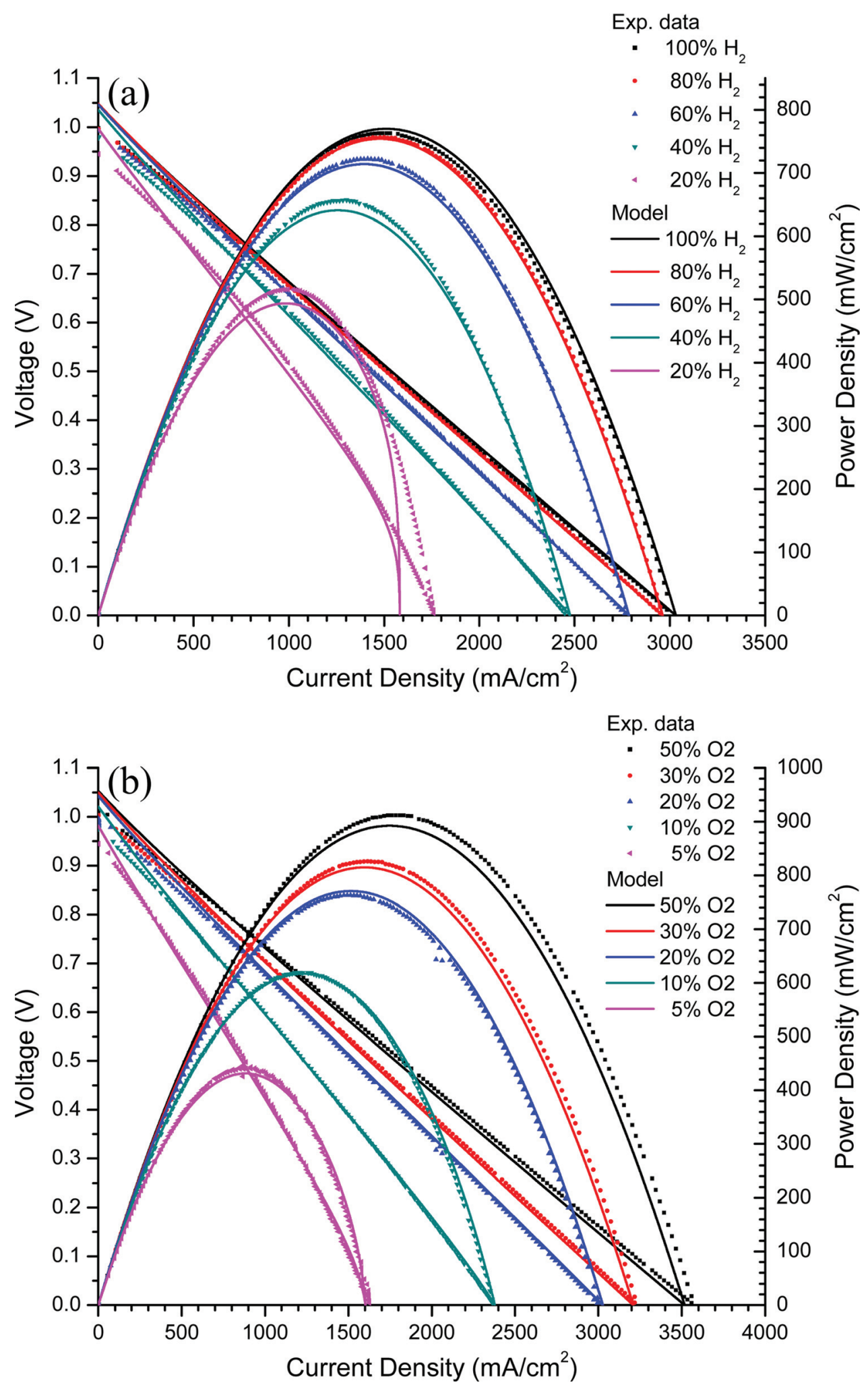

Figure 3. (Color online) Comparison of experimental results for $V(i)$ with our model predictions for different (a) $\mathrm{H}_{2}$ partial pressures of the anode inflow (using air for the cathode) and (b) $\mathrm{O}_{2}$ partial pressures at the cathode inflow (using $100 \% \mathrm{H}_{2}$ for the anode). electronic conductivity of the electrolyte and results in lower OCV. ${ }^{2-6}$ The electronic conductivity of $\mathrm{Ba}\left(\mathrm{Ce}_{0.8} \mathrm{Y}_{0.2}\right) \mathrm{O}_{3-\delta}$ increases rapidly with increasing operating temperature, which explains the bigger voltage difference of OCV between the theoretical emf and measured $\mathrm{OCV}$ at $800^{\circ} \mathrm{C}$ than $700^{\circ} \mathrm{C}$.

The $\mathrm{H}_{2}$ partial-pressure-dependent $i$ - $V$ curves while air was used on the cathode side at $700^{\circ} \mathrm{C}$ are shown on Fig. 3a. The convex $i-V$ curve of $20 \% \mathrm{H}_{2}$ indicates concentration polarization came into play when high current density was driven from the cell. The peak power outputs increased dramatically from 20 to $60 \% \mathrm{H}_{2}$ partial pressure but saturated when higher $\mathrm{H}_{2}$ partial pressure gases was used, with almost no change between 80 and $100 \% \mathrm{H}_{2}$.

A series of oxygen partial pressure dependent tests at the cathode side with $100 \% \mathrm{H}_{2}$ as anode fuel gas is shown on Fig. 3b. It is 
surprising that the cell can be run with $\mathrm{O}_{2}$ partial pressure as low as $5 \%$ without encountering serious cathodic polarization. This polarization can be referred to as cathodic activation polarization since the cathode is so thin that the oxygen partial pressure across the cathode can remain constant as current density changes. A voltage drop would be called cathodic concentration polarization only if oxygen partial pressure drops across the cathode when the current density is increasing. The peak power outputs clearly increase with increasing $\mathrm{O}_{2}$ partial pressure. However, if it is economically efficient to use high $\mathrm{O}_{2}$ concentration gas instead of air to run the system, the producing of steam on the cathode side of the H-SOFC would require removing this steam if the unused oxygen is recirculated.

Compared to our previous report, ${ }^{7}$ the cell performance here is much better even with thicker electrolyte. Several reasons that can lead to the thicker electrolyte cell having better performance include lower tortuosity of supporting anode, better sintering temperature for the cathode and using of silver paste on the cathode surface. The major reason for this result was the use of silver paste. Different tests, not shown in both papers, showed much better performances when silver paste was used on the cathode. Two possible reasons may explain why silver helps the performance. First, the silver reduces the cathode ohmic resistance since silver is a good conductor. Second, silver helps the oxygen dissociation on the cathode material and increases the cell performance. The effect of using silver paste is beyond the goal of this paper and also needs further study.

\section{Discussion}

Basics of H-SOFC voltage contribution. - To simplify the modeling, we idealize the H-SOFC as having a solid electrolyte that is a pure proton conductor, and an anode that is a pure electronic conductor. The cathode, LSM in our experiment, is also assumed to be a pure electronic conductor, and it is further assumed that oxygen molecules can dissociate on its surface and then neutral $\mathrm{O}$ atoms can diffuse along the surface to the TPB.

The open-circuit emf $V_{0}$ arises at the anode-electrolyte and cathode-electrolyte interfaces where electrochemical reactions occur. There is a third type of site, on the pore-cathode interface, where oxygen molecules adsorb and dissociate into oxygen atoms that diffuse along this interface to the TPB. However, there cannot be an emf difference across this pore-cathode interface because the pore is an insulator. For closed-circuit conditions, the three additional contributions besides $V_{0}$ to terminal voltage $V$ are commonly called activation polarization $V_{a c t}$, concentration polarization $V_{c o n c}$ and ohmic polarization $V_{\text {ohm }}$. These three are taken as positive in the SOFC mode. They become negative in the solid oxide electrolysis cell (SOEC) mode for which $i$ flow in the opposite direction. Then in both the SOFC and SOEC modes, the terminal voltage is given by

$$
V=V_{0}-V_{a c t}-V_{c o n c}-V_{o h m}
$$

In the SOFC mode, $V$ is output voltage, while in the SOEC mode, $V$ is applied voltage.

For the open-circuit case, at the two interfaces, the forward reaction rates are balanced by the reverse reaction rates. There is an equivalent current density for each reaction, which is the product of an attempt current density and a success probability. Thermodynamically, the attempt current densities are related to entropy and success probabilities to enthalpy and to voltage steps at the TPB. For closed-circuit operation, even if the gas concentrations at the interfaces remain unchanged, the forward and reverse reaction rates must become imbalanced to allow net current to flow. This imbalance is achieved by altering the success probability by means of changing the potential step at each interface. This change in potential step is called the activation polarization for each interface. The additional change in potential step if gas concentrations at the interface alter when current flows is called the concentration polarization for that interface. Finally, the ohmic polarization is the ohmic volt-

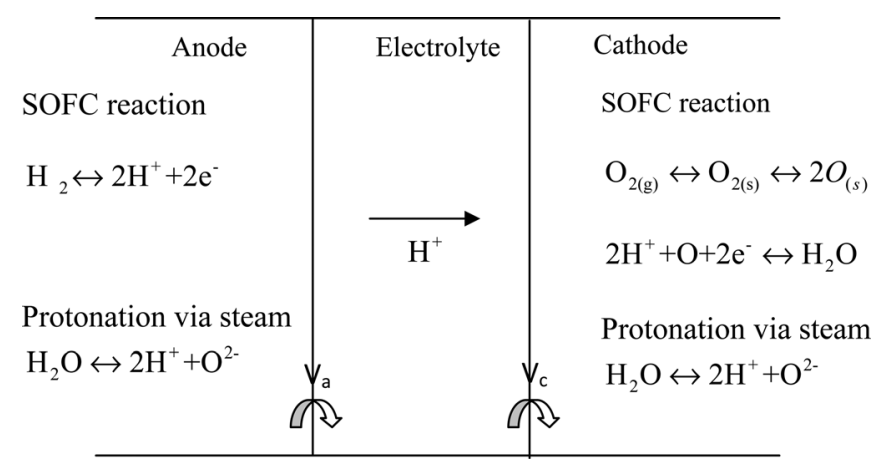

Figure 4. Electrochemical reactions for an H-SOFC.

age drop due to the transfer of ions across the electrolyte and electrons across the anode, cathode and electrolyte. The drop is mainly distributed across the electrolyte, with weaker drops across the anode and cathode.

Model for reactions and their equivalent current density contributions. - Two of the three reversible reactions which contribute to SOFC or SOEC output occur at the anode-electrolyte and cathode-electrolyte interfaces. They are assumed to occur directly at the triple phase boundary (TPB) sites, located on lines where pore, electrolyte and electrode meet. The third reaction, oxygen adsorption and dissociation, takes place on the surface where pore and cathode material meet. Of these three reactions for the H-SOFC with $\mathrm{H}_{2}$ fuel and $\mathrm{O}_{2}$ oxidizer, only the first two reactions include electron transfer.

Additional reactions occur that do not contribute electrons to the external circuit, but contribute to protonation or deprotonation of the electrolyte. One of these reactions, protonation via steam, uses the steam produced in the SOFC mode, or any steam otherwise present at the anode and/or cathode, to provide protons for the proton conduction mechanism, Fig. 4. This protonation mechanism requires at least a small amount of oxygen ion conductivity in the electrolyte, in addition to the dominant protonic conduction. After equilibrium proton concentration is achieved, this reaction can still transfer steam across the electrolyte. For instance, even for open circuit if there is steam supplied only to the cathode, a combination of conduction and diffusion of protons and oxygen ions across the electrolyte will transfer steam to the anode. An alternate protonation mechanism based on the $\mathrm{H}_{2} \rightarrow 2 \mathrm{H}^{+}+2 e^{-}$reaction at the anode interface, occurs as a net reaction only during protonation and not after equilibrium is reached, and cannot contribute to steam transfer across the electrolyte. This reaction requires at least a small amount of electronic conductivity. Accordingly, protonation requires either some oxygen ion or electronic conductivity in the electrolyte to maintain charge neutrality across the electrolyte, but only some oxygen ion conductivity can account for steam creation at the anode interface.

For the SOFC reactions at the cathode, we propose a three-step mechanism, i.e. a two-step oxygen adsorption reaction, $O_{2(g)} \leftrightarrow O_{2(s)} \leftrightarrow 2 O_{(s)}$, at the pore-cathode interface, where $O_{(s)}$ signifies an $\mathrm{O}$ atom adsorbed on the cathode surface, and a reaction $2 \mathrm{H}^{+}+\mathrm{O}_{(s)}+2 e^{-} \leftrightarrow \mathrm{H}_{2} \mathrm{O}_{(g)}$ at the cathode-electrolyte TPBs. A one-step cathode reaction, i.e. $4 \mathrm{H}^{+}+\mathrm{O}_{2}+4 e^{-} \leftrightarrow 2 \mathrm{H}_{2} \mathrm{O}$, cannot be dominant because an $\mathrm{O}_{2}$ molecule is required to react with four protons and four electrons at a cathode TPB site at the same time to form $2 \mathrm{H}_{2} \mathrm{O}$ molecules. The concentration of protons in the electrolyte is low $(<20 \%)$, so the probability of four protons simultaneously being within reaction distance is too low to provide the dominant reaction mechanism. For the oxygen adsorption reaction, $\mathrm{O}_{2}$ molecules first adsorb onto the surface of the cathode ceramic and subsequently dissociate into $\mathrm{O}$ atoms on the ceramic surface

$$
O_{2(g)} \leftrightarrow O_{2(s)} \leftrightarrow 2 O_{(s)}
$$


We will model this two-step reaction as a single reaction. This reaction does not need to be on a TPB; it can occur anywhere on the cathode-pore interface since no electron transfer is involved. The other reaction at the cathode is

$$
2 H^{+}+O_{(s)}+2 e^{-} \leftrightarrow H_{2} O_{(g)}
$$

We assume that the electrolyte is a pure proton conductor, so this reaction must occur at the cathode-electrolyte TPBs.

Reaction at anode side.- We first model the reaction at the anode-electrolyte TPBs. The reaction is

$$
H_{2} \leftrightarrow 2 H^{+}+2 e^{-}
$$

Consider a line of oxygen ion sites in the electrolyte along a TPB line. We assume $v_{\text {ele }}$ is the oxygen vacancy probability that the $O^{2-}$ site is vacant in the electrolyte, and so $1-v_{\text {ele }}$ is the probability that the $O^{2-}$ site is occupied. We designate $v_{a f}$ as the anode forward reaction rate, the probability per unit time that the forward reaction in Eq. 4 will occur at each occupied site. Similarly, we designate $v_{a r}$ as anode reverse reaction rate.

One factor in $v_{a f}$ is the attempt rate, the probability per unit time $v_{a f 0}$ that an $\mathrm{H}_{2}$ molecule will strike a given occupied $O^{2-}$ site and attempt to cause a reaction. Another factor is success probability that the forward reaction of Eq. 4 will occur when the $\mathrm{H}_{2}$ molecule strikes the $O^{2-}$ site. If the reaction occurs, it will release heat $U_{a}$ less the energy $q V_{a}$ required for two electrons of combined charge magnitude $q$ to travel across the potential difference $V_{a}$ between anode and electrolyte. Thus, the net energy required for the reaction to occur is $q V_{a}-U_{a}$. If the reaction does not occur, the $\mathrm{H}_{2}$ molecule bounces back into the anode pore.

Accordingly, the attempt rate is for a collision event that may or may not lead to a reaction. Assume the probability of either a reaction or no reaction can be described by classical (Boltzmann) statistics. The un-normalized probability for the reaction event is $\exp \left[-\left(q V_{a}-U_{a}\right) / k T\right]$ and for no reaction event is one. Normalizing, and multiplying by $v_{a f 0}$, we obtain the reaction rate per $O^{2-}$ site

$$
v_{a f}=v_{a f 0} \exp \left[-\left(q V_{a}-U_{a}\right) / 2 k T\right] / 2 \cosh \left[\left(q V_{a}-U_{a}\right) / 2 k T\right]
$$

A similar expression, but with opposite sign for the argument of the exponential, holds for the reverse reaction based on recombining two protons in the electrolyte to form an $\mathrm{H}_{2}$ molecule

$$
v_{a r}=v_{a r 0} \exp \left[\left(q V_{a}-U_{a}\right) / 2 k T\right] / 2 \cosh \left[\left(q V_{a}-U_{a}\right) / 2 k T\right]
$$

To find $v_{a f 0}$, we consider an $\mathrm{H}_{2}$ fuel gas concentration of $n_{H_{2}}$ at the anode-electrolyte interface. Half of these $\mathrm{H}_{2}$ molecules travel toward the surface with mean normal velocity component $\left\langle u_{y}\right\rangle$. Then the flux of $\mathrm{H}_{2}$ molecules onto the surface will be $\frac{1}{2} n_{H_{2}}\left\langle u_{y}\right\rangle$. We know $\frac{1}{2} k T=\frac{1}{2} m_{H_{2}}\left\langle u_{y}^{2}\right\rangle$, so then $\left\langle u_{y}\right\rangle=\left(k T / m_{H_{2}}\right)^{1 / 2}$, where $m_{H_{2}}$ is the mass of the $\mathrm{H}_{2}$ molecule. Thus, the flux is $\frac{1}{2} n_{\mathrm{H}_{2}}\left(k T / m_{\mathrm{H}_{2}}\right)^{1 / 2}$. The perovskite structure $\left(\mathrm{ABO}_{3}\right)$ has three $\mathrm{O}^{2-}$ sites in each unit cell. Accordingly, as an approximation for the surface density of $O^{2-}$ reaction sites, we take the $2 / 3$ power of the volume density, namely $\left(3 / a_{\text {ele }}^{3}\right)^{2 / 3}=3^{2 / 3} / a_{\text {ele }}^{2}$, where $a_{\text {ele }}$ is the perovskite lattice constant, $0.4445 \mathrm{~nm}$ for $\mathrm{Ba}\left(\mathrm{Ce}_{0.8} \mathrm{Y}_{0.2}\right) \mathrm{O}_{3-\delta}$. We assume that all points on the surface are close enough to an $O^{2-}$ site for a reaction to occur. Then, each $O^{2-}$ site has reaction area $a_{\text {ele }}^{2} / 3^{2 / 3} \mathrm{~m}^{2}$ and the impingement rate per $O^{2-}$ site is

$$
v_{a f 0}=\frac{1}{2} n_{H_{2}}\left(\frac{k T}{m_{H_{2}}}\right)^{1 / 2}\left(\frac{a_{\text {ele }}^{2}}{3^{2 / 3}}\right)
$$

Only a fraction $f_{a}$, to be estimated later, of oxygen sites sit on the TPB and are available for the reactions at the anode-electrolyte TPB.

To further analyze the reaction of Eq. 4, we need to make assumptions about the nature of protonic location and conductivity in the perovskite structure. Two clues come from the two Pauling ice rules. We consider them because the $O-O$ separation in perovskites is similar to that in ice. Thus the protons should reside in offcenter positions in $O-H \cdots O \mathrm{H}$-bonds in perovskites, as they do in ice. Pauling's first rule is that there can be one and only one proton in each possible $\mathrm{H}$-bond. In perovskites there are way too few protons to fill the possible $\mathrm{H}$-bonds, so a proton has no problem finding an adjacent vacant bond to transfer to, as one of the steps in protonic conduction. The other step is proton intrabond transfer, $\mathrm{O}-\mathrm{H} \cdots \mathrm{O} \rightarrow \mathrm{O} \cdots \mathrm{H}-\mathrm{O}$.

Pauling's second rule is that two protons are close to a given oxygen, and two farther away, in the four asymmetric $\mathrm{H}$-bonds radiating out from that oxygen. Accordingly, individual $\mathrm{H}_{2} \mathrm{O}$ "molecules" can be identified in the ice structure. Thus in the Eq. 4 reaction we expect that such an $\mathrm{H}_{2} \mathrm{O}$ "molecule" involving one oxygen and two $\mathrm{H}$-bonds are formed.

We now analyze the hydrogen dissociation reaction of Eq. 4 . We need an oxygen ion, which has no proton attached to it, for this dissociation of the $\mathrm{H}_{2}$ molecule to occur. The probability that an $\mathrm{O}^{2-}$ site is occupied by an oxygen ion is $1-v_{\text {ele }}$. In the perovskite structure, each oxygen ion has a volume $a_{\text {ele }}^{3} / 3$ associated with it. If the proton concentration in the electrolyte is $n_{H, e l e}$, then per oxygen site the joint probability of an $O^{2-}$ ion with one or two protons attached to it is approximately $\left(1-v_{\text {ele }}\right)\left(\frac{a_{\text {ele }}^{3} n_{H, e l e}}{3}+\frac{a_{\text {ele }}^{6} n_{H, e l e}^{2}}{9}\right)$, and $\left(1-v_{\text {ele }}\right)\left(1-\frac{a_{\text {ele }}^{3} n_{H, \text { ele }}}{3}-\frac{a_{\text {ele }}^{6} n_{H, \text { ele }}^{2}}{9}\right)$ for an $O^{2-}$ ion without any proton attached to it. The current densities are related by a factor $q$ to the gas flow densities resulting in the reaction. By defining $\alpha \equiv\left(U_{a}-q V_{a}\right) / 2 k T$ and putting all the factors together, we obtain

$$
\begin{aligned}
i_{a f}= & \frac{1}{2} n_{H_{2}}\left(\frac{k T}{m_{H_{2}}}\right)^{1 / 2}\left(\frac{a_{\text {ele }}^{2}}{3^{2 / 3}}\right) \times f_{a} \times\left(\frac{3^{2 / 3}}{a_{\text {ele }}^{2}}\right) \times\left(1-v_{\text {ele }}\right) \\
& \times\left[1-\left(\frac{a_{\text {ele }}^{3} n_{H, \text { ele }}}{3}\right)-\left(\frac{a_{\text {ele }}^{6} n_{H, \text { ele }}^{2}}{9}\right)\right] \times q \times e^{\alpha} / 2 \cosh \alpha \\
= & \frac{1}{2} n_{H_{2}}\left(\frac{k T}{m_{H_{2}}}\right)^{1 / 2} f_{a} q\left(1-v_{\text {ele }}\right)\left[1-\left(\frac{a_{\text {ele }}^{3} n_{H, \text { ele }}}{3}\right)-\left(\frac{a_{\text {ele }}^{6} n_{H, \text { ele }}^{2}}{9}\right)\right] \\
& \times e^{\alpha} / 2 \cosh \alpha \\
= & a e^{\alpha} / 2 \cosh \alpha, \quad \text { where } a \equiv \frac{1}{2} n_{H_{2}}\left(\frac{k T}{m_{H_{2}}}\right)^{1 / 2} f_{a} q\left(1-v_{\text {ele }}\right) \\
& \times\left[1-\left(\frac{a_{\text {ele }}^{3} n_{H, \text { ele }}}{3}\right)-\left(\frac{a_{\text {ele }}^{6} n_{H, \text { ele }}^{2}}{9}\right)\right]
\end{aligned}
$$

For the reverse reaction to be possible there must be two close protons in the electrolyte in the volume for one oxygen ion, which has a probability $a_{\text {ele }}^{6} n_{H, \text { ele }}^{2} / 9$. Therefore, the density of oxygen ions with two protons attached to them is

$$
\frac{3^{2 / 3}\left(1-v_{\text {ele }}\right)}{a_{\text {ele }}^{2}} \times \frac{a_{\text {ele }}^{6} n_{H, \text { ele }}^{2}}{9}=3^{2 / 3}\left(1-v_{\text {ele }}\right) a_{\text {ele }}^{4} n_{H, \text { ele }}^{2} / 9
$$

We consider a given reaction attempt is the probability that the reaction occurs during a single period of a typical lattice vibration frequency $v$. Above the Debye temperature $\Theta$ this frequency is approximately equal to $k \Theta / h$, where $h$ is Planck's constant. If $\Theta$ is not known, it is common practice to use $v=10^{13} / \mathrm{sec}$ as an approximation. This reverse reaction is also located on the TPB lines, so

$$
\begin{aligned}
i_{a r} & =\left(3^{2 / 3}\left(1-v_{\text {ele }}\right) a_{\text {ele }}^{4} n_{H, \text { ele }}^{2} / 9\right) f_{a} \cup q e^{-\alpha} / 2 \cosh \alpha \\
& =b e^{-\alpha} / 2 \cosh \alpha, \quad \text { where } b \equiv 3^{2 / 3}\left(1-v_{\text {ele }}\right) a_{\text {ele }}^{4} n_{H, \text { ele }}^{2} f_{a} \vee q / 9
\end{aligned}
$$

Here $a$ and $b$ are the anode attempt current densities. For open circuit, we designate $a, b, i_{a f}$, and $i_{a r}$ with subscript 0 . The forward 
and reverse open-circuit reaction rates are equal because $i$ $=i_{a f 0}-i_{a r 0}=0$ in the electrolyte. These equivalent equal and opposite forward and reverse current densities are commonly designated as $i_{0}$, the exchange current density. In general, $i_{0}$ has different values for the reactions at the anode-electrolyte TPB, $i_{0 a}$, and the cathode-electrolyte TPB, $i_{0 c}$. To find exchange current density $i_{0 a}$ at the anode, we know that

$$
i_{a f 0}=i_{a r 0}=i_{0 a}
$$

From Eqs. 8 and 10 for the open-circuit case, together with Eq. 11, we have

$$
a_{0} \exp \left(\alpha_{0}\right)=b_{0} \exp \left(-\alpha_{0}\right)=2 i_{0 a} \cosh \left(\alpha_{0}\right)
$$

From Eq. 12 we obtain

$$
\alpha_{0} \equiv \frac{1}{2} \ln \left(b_{0} / a_{0}\right)
$$

For open circuit, the $\mathrm{H}_{2}$ concentration $n_{H_{2}}$ at the anode-electrolyte TPB is the same as at the outside plenum and the value $V_{a 0}$ across the anode-electrolyte interface is the contribution to the open-circuit SOFC emf $V_{0}$.

Reactions at cathode side.- As discussed previously, there are two reactions at the cathode side, one at the cathode-electrolyte TPB and the other at the cathode-pore interface. Similar to the arguments for the reaction at the anode side, both reactions on the cathode side are treated as collision events. Looking at a (001) surface of the perovskite structure, one could assume that the $\mathrm{O}$ atom sits in a square surface "pocket" of surface area $a_{c}^{2} / 2$ bounded by 2 A-site cations and $2 \mathrm{O}^{2-}$ ions, where $a_{c}$ is the LSM cathode lattice constant. There are four adjacent such pockets which could hold another $\mathrm{O}$ atom. We designate $n_{O}$ as the $\mathrm{O}$ atom concentration on the cathode material surface. The probability for two empty sites next to each other is $\left(1-\frac{1}{2} n_{O} a_{c}^{2}\right)^{2}$. After taking into account the impinging rate, the attempt rate per unit ceramic surface area for Eq. $2 O_{2(g)} \leftrightarrow$ $O_{2(s)} \leftrightarrow 2 O_{(s)}$, is

$$
\frac{1}{2} n_{O_{2}}\left(\frac{k T}{m_{O_{2}}}\right)\left(1-\frac{1}{2} n_{O} a_{c}^{2}\right)^{2} \frac{a_{c}^{2}}{2}
$$

and the forward reaction rate is

$$
\mathrm{v}_{\text {cof }}=\frac{1}{2} n_{O_{2}}\left(\frac{k T}{m_{O_{2}}}\right)\left(1-\frac{1}{2} n_{O} a_{c}^{2}\right)^{2} e^{\left(U_{d} / 2 k T\right)} / 2 \cosh \left(U_{d} / 2 k T\right)
$$

where $n_{O_{2}}$ is the $O_{2}$ concentration in the cathode pore, $m_{O_{2}}$ is the molecular mass of $\mathrm{O}_{2}$, and $U_{d}$ is the enthalpy released in the adsorption/dissociation combined reaction.

For the reverse reaction, we need two adjacent $\mathrm{O}$ atoms for recombination into an $\mathrm{O}_{2}$ molecule on the surface of the cathode material. There are four adjacent such pockets which could hold another $\mathrm{O}$ atom as possible recombination partner for a given pocket. To avoid double counting of the recombination attempts, a factor of $1 / 2$ needs to be taken into account. Based on the assumptions above, we have the attempt rate for reverse reaction

$$
v n_{O}^{2} a_{c}^{2}
$$

and the reverse reaction rate

$$
v_{c o r}=v n_{O}^{2} a_{c}^{2} e^{\left(-U_{d} / 2 k T\right)} / 2 \cosh \left(U_{d} / 2 k T\right)
$$

Based on setting the forward and reverse reaction probabilities equal in equilibrium, and assuming that decrease (for SOFC mode) or increase (for SOEC mode) of $\mathrm{O}$ atom concentration from reactions with protons and electrons to form or dissociate $\mathrm{H}_{2} \mathrm{O}$ is negligible, we can find the $\mathrm{O}$ atom concentration on the surface of cathode material to be

$$
n_{O}=\frac{\varsigma}{\left(\frac{1}{2} a_{c}^{2} \varsigma+1\right)} \text { where } \varsigma=\left(\frac{n_{O_{2}}}{2 v}\right)^{1 / 2}\left(\frac{k T}{m_{O_{2}}}\right)^{1 / 4} a_{c}^{-1} e^{\left(U_{d} / 2 k T\right)}
$$

Now we look at the reaction on the TPB at the cathode-electrolyte interface, $2 \mathrm{H}^{+}+\mathrm{O}_{(s)}+2 e^{-} \leftrightarrow \mathrm{H}_{2} \mathrm{O}_{(g)}$. The forward and reverse current densities are again the product of an attempt rate and a success probability. Thus

$$
\begin{aligned}
& i_{c f}=c e^{\beta} / 2 \cosh \beta \text { and } \\
& i_{c r}=d e^{-\beta} / 2 \cosh \beta, \quad \text { where } \beta \equiv\left(U_{c}-q V_{c}\right) / 2 k T
\end{aligned}
$$

Here $V_{c}$ is the potential difference across the cathode-electrolyte interface, and the reaction releases heat $U_{c}$ when the forward reaction occurs. Thus the net energy required for the forward reaction of Eq. 3 to occur is $q V_{c}-U_{c}$. We assume that this forward reaction can occur only if two protons are adjacent to the same oxygen ion, in order to be close enough together to participate in the forward reaction. The probability that a given $O^{2-}$ ion has two adjacent protons is $a_{e l e}^{6} n_{H, e l e}^{2} / 9$. The $O^{2-}$ ion concentration available at the TPB from the electrolyte is $\frac{3^{2 / 3}}{a^{2}}\left(1-v_{\text {ele }}\right) f_{c}$ in which $\left(1-v_{\text {ele }}\right)$ is the probability that the $O^{2-}$ site is occupied and $f_{c}$ is the probability of the $\mathrm{O}^{2-}$ site being at a TPB on the cathode side. Assuming that there is only one $\mathrm{O}$ atom site in the cathode adjacent to the site holding the two protons, we saw above that the probability of an $\mathrm{O}$ atom occupying that site is $n_{O} a_{c}^{2} / 2$. Putting all these factors together and multiplying the transferred charge $q$, attempt frequency $v$ and success probability, the cathode forward reaction current density is

$$
\begin{aligned}
i_{c f} & =\frac{3^{2 / 3} n_{O} a_{c}^{2} a_{\text {ele }}^{4} n_{H, \text { ele }}^{2}\left(1-v_{\text {ele }}\right) f_{c} q v}{18} e^{\beta} / 2 \cosh \beta \\
& =c e^{\beta} / 2 \cosh \beta, \quad \text { where } c \equiv \frac{3^{2 / 3} n_{O} a_{c}^{2} a_{\text {ele }}^{4} n_{H, \text { ele }}^{2}\left(1-v_{\text {ele }}\right) f_{c} q v}{18}
\end{aligned}
$$

To calculate the current density of the reverse reaction of Eq. 3, we assume the steam concentration on the cathode-electrolyte TPB is $n_{c, \mathrm{H}_{2} \mathrm{O}}$. The reaction needs an oxygen ion on the electrolyte surface, which has no proton attached to it. The probability that the oxygen ion site fulfils these conditions is $\left(1-v_{\text {ele }}\right)\left[1-\left(\frac{a_{\text {ele }}^{3} n_{\text {Hele }}}{3}\right)\right.$ $\left.-\left(\frac{a_{e l e}^{6} n_{H, e l e}^{2}}{9}\right)\right]$. The reaction also needs an adjacent vacant $\mathrm{O}$ atom site on the cathode surface, and the probability that the site is vacant is $1-\frac{1}{2} n_{O} a_{c}^{2}$. The $O^{2-}$ ion from the electrolyte and the $\mathrm{O}$ atom site from the cathode need to be on the cathode-electrolyte TPB for the reaction. We also need to consider the impinging rate of water molecules and the charge transfer per reaction. We obtain

$$
\begin{aligned}
i_{c r}= & \frac{1}{2} n_{c, H_{2} O}\left(\frac{k T}{m_{H_{2} O}}\right)^{1 / 2}\left[1-\left(\frac{a_{\text {ele }}^{3} n_{H, \text { ele }}}{3}\right)-\left(\frac{a_{\text {ele }}^{6} n_{H, \text { ele }}^{2}}{9}\right)\right] \\
& \times\left(1-v_{\text {ele }}\right)\left(1-\frac{n_{O} a_{c}^{2}}{2}\right) f_{c} q e^{-\beta} / 2 \cosh \beta \\
= & d e^{-\beta / 2} \cosh \beta
\end{aligned}
$$

where $\quad d \equiv \frac{1}{2} n_{c, H_{2} O} O\left(\frac{k T}{m_{H_{2} O} O}\right)^{1 / 2}\left[1-\left(\frac{a_{\text {ele }}^{3} n_{H, \text { ele }}}{3}\right)-\left(\frac{a_{\text {ele }}^{6} n_{H, \text { ele }}^{2}}{9}\right)\right]\left(1-v_{\text {ele }}\right)$ $\times\left(1-\frac{n_{o} a_{c}^{2}}{2}\right) f_{c} q$.

In Eqs. 20 and 21, $c$ and $d$ are the forward and reverse attempt current densities for the reaction at the cathode, respectively.

Derivation of current density as a function of activation polarization.- To introduce some ideas needed to derive our $V(i)$ expression, we first find $i$ as a function of anode activation 
polarization $V_{a, a c t}$ or cathode activation polarization $V_{c, a c t}$, neglecting concentration and ohmic polarization effects. To be specific, we study the anode case and take the open-circuit attempt current densities as $a_{0}$ and $b_{0}$. We begin with $i=i_{a f}-i_{a r}$ and Eqs. 8 and 10 and use the relation $\alpha=\alpha_{0}+\delta$, where $\alpha_{0}$ is the open-circuit value and $\delta=q V_{\text {a,act }} / 2 k T$ is the change in $\alpha$ needed to get nonzero $i$. We have

$i=i_{a f}-i_{a r}=\left[a_{0} \exp \left(\alpha_{0}+\delta\right)-b_{0} \exp \left(-\alpha_{0}-\delta\right)\right] / 2 \cosh \left(\alpha_{0}+\delta\right)$

We know that $a_{0} \exp \left(\alpha_{0}\right)=b_{0} \exp \left(-\alpha_{0}\right)$ from Eq. 12 and $\cosh \left(\alpha_{0}+\delta\right)=\cosh \alpha_{0} \cosh \delta+\sinh \alpha_{0} \sinh \delta$. We obtain

$$
\begin{aligned}
i & =a_{0} \exp \left(\alpha_{0}\right)[\exp \delta-\exp (-\delta)] / 2\left[\cosh \alpha_{0} \cosh \delta+\sinh \alpha_{0} \sinh \delta\right] \\
& =a_{0} \exp \left(\alpha_{0}\right) \tanh \delta /\left[\cosh \alpha_{0}+\sinh \alpha_{0} \tanh \delta\right]
\end{aligned}
$$

From Eq. 12, we know that $2 i_{0 a}=a_{0} \exp \left(\alpha_{0}\right) / \cosh \alpha_{0}$, so

$$
i=2 i_{0 a} \tanh \delta /\left[1+\tanh \alpha_{0} \tanh \delta\right]
$$

To find tanh $\alpha_{0}$, we start by rearranging Eqs. 8 and 10 and using $i=i_{a f}-i_{a r}$,

$$
i=\frac{1}{2}(a-b)+\frac{1}{2}(a+b) \tanh \alpha
$$

For our present $V_{\text {conc }}=0$ case, $a$ and $b$ have subscripts 0 . From Eqs. 12 and 13 , we obtain

$$
i_{0 a}=\left(a_{0}^{-1}+b_{0}^{-1}\right)^{-1}
$$

and to find $\tanh \left(\alpha_{0}\right)$ we set $i=0$ in Eq. 25 and get

$$
\tanh \left(\alpha_{0}\right)=\left(b_{0}-a_{0}\right) /\left(b_{0}+a_{0}\right)=\left(a_{0}^{-1}-b_{0}^{-1}\right) i_{0 a}
$$

Then Eq. 25 can be written in the form

$$
i=2 i_{0 a} \tanh \delta /\left[1+\left(a_{0}^{-1}-b_{0}^{-1}\right) i_{0 a} \tanh \delta\right]
$$

This differs from Butler-Volmer expressions seen in the Refs. 8-13 partly because the finite rate of fuel gas molecule impingement is included in the derivation. Examples of these differences are now shown for two papers. First, Zhu et al. ${ }^{12}$ in their Appendix derive Butler-Volmer expressions for both the anode and cathode reactions. The anode expression in their Eq. A15 does not have charge transfer coefficient 0.5 . However, the cathode expression in Eq. A28 does have the commonly-used coefficient 0.5 after they assume $\beta_{44 . a}=\beta_{44, c}=1 / 2$, thus allowing the sinh form $i=2 i_{0} \sinh \left(F \eta_{c} / 2 R T\right)$. Presumably their exchange current density $i_{0}$ varies with cathode activation overpotential $\eta_{c}$ to keep $i$ from unphysically going to infinity as $\eta_{c}$ becomes infinite. Second, Bessler et al. ${ }^{13}$ in their Eq. 1 seem to avoid this unphysical result by specifically including a $P_{O 2}^{a}$ factor that presumably varies with $\eta$ in their Butler-Volmer expression $i=2 i^{0} p_{O 2}^{a} \exp \left(-E_{a c t} / R T\right)$ sinh $(0.5 F \eta / R T)$. The $\exp \left(-E_{a c t} / R T\right)$ factor allows for a charge transfer coefficient different from 0.5 , and also can allow for a reaction barrier over and above the electrochemical energy step required for the reaction. In our present work we assume a coefficient 0.5 and no reaction barrier.

To obtain limiting case values for $i$, we note that in the SOFC mode, when $V_{a c t} \rightarrow \infty,\left(V_{a} \rightarrow-\infty\right.$, the impractical case of applying a large negative voltage) $\tanh \alpha \rightarrow 1$. From Eq. 25 the current density $i$ then reaches $a_{0}$. This value makes sense physically because $a_{0}$ corresponds to the rate at which all $\mathrm{H}_{2}$ molecules striking on the TPB with $O^{2-}$ ions present dissociate into protons. In the SOEC electrolysis mode, for high positive applied voltage, current density will be limited to $i=-b_{0}$. This value corresponds to all protons in the electrolyte along the TPB recombining into $\mathrm{H}_{2}$ molecules, i.e. maximum $\mathrm{H}_{2}$ production rate when running the cell as an SOEC.

Derivation of voltage as a function of current density.- To derive $V(i)$ for SOFC and SOEC, we need to include the contribu- tion from all polarizations, i.e. $V=V_{0}-V_{a c t}-V_{\text {cenc }}-V_{\text {ohm }}$ We first consider the anode-electrolyte TPB, which contributes $V_{a}$ to the terminal voltage $V$. Rearranging Eq. 25 and noting that it is valid also when both activation and concentration polarizations are present, we have

$$
\tanh \alpha=(2 i-a+b) /(a+b)
$$

In general, we know that $\tanh ^{-1} x=\frac{1}{2} \ln (1+x)-\frac{1}{2} \ln (1-x)$, so

$$
\alpha=\frac{1}{2} \ln [(b+i) /(a-i)]=\left(U_{a}-q V_{a}\right) / 2 k T
$$

Solving Eq. 30 for $V_{a}$ gives

$$
V_{a}=U_{a} / q-(k T / q) \ln [(b+i) /(a-i)]
$$

Similarly, $V_{c}$ can be found from Eqs. 19, 20 and 21 as

$$
V_{c}=\left(U_{c} / q\right)-(k T / q) \ln [(d+i) /(c-i)]
$$

Adding Eqs. 31 and 32 gives the terminal voltage $V(i)$ for the cell

$$
\begin{aligned}
V(i)= & V_{a}+V_{c}=\left(U_{a}+U_{c}\right) / q-(k T / q) \\
& \times \ln [(b+i)(d+i) /(a-i)(c-i)]
\end{aligned}
$$

A difficulty in interpreting the relation in that form is that the enthalpy released by the entire forward reaction for the fuel cell is not $U_{a}+U_{c}$, but rather is $U_{a}+U_{c}+\frac{1}{2} U_{d} \equiv U$. This difficulty can be solved mathematically by writing $V$ as

$$
V(i)=U / q-(k T / q) \ln \left[\frac{(b+i)(d+i)}{(a-i)(c-i)} e^{\left(U_{d} / 2 k T\right)}\right]
$$

What if the oxygen is supplied for the cathode reaction by means of surface diffusion by oxygen ions instead of oxygen atoms? There will still be a potential step at the cathode-electrolyte TPB that the protons must overcome to participate in the reaction. Although $U_{c}$ and $U_{d}$ will be different, the sum of $U_{c}+\frac{1}{2} U_{d}$ must remain the same. Accordingly, the only changes will be that Eq. 2 will become

$$
O_{2(g)} \leftrightarrow O_{2(s)} \leftrightarrow O_{2(s)}+4 e^{-} \leftrightarrow 2 O_{(s)}^{2-}
$$

Eq. 3 will become

$$
2 \mathrm{H}^{+}+\mathrm{O}_{(s)}^{2-} \leftrightarrow \mathrm{H}_{2} \mathrm{O}_{(g)}
$$

Protonic and electronic conductivity in H-SOFC.- If electronic conductivity occurs in the electrolyte, Eq. 33 gives $V\left(i_{p}\right)$ instead of $V(i)$. We expect that the voltage steps $V_{a}$ and $V_{c}$ depend only on the proton current in the electrolyte, $i_{p}$, and not on electronic current in the electrolyte, $i_{e}$. Then, in the usual expression for terminal voltage

$$
V=V_{0}-V_{a c t}-V_{c o n}-V_{o h m}
$$

$V_{0}$ is still the open-circuit voltage based on thermodynamic calculation, whereas the other three contributions are functions of $i_{p}$. In the model, we know the $V_{a c t}\left(i_{p}\right)$ and $V_{c o n}\left(i_{p}\right)$ contributions for both the anode and cathode sides. We assume that in the interior of the electrolyte there are no unbalanced charges, so that the field $\mathrm{E}$ is uniform across the electrolyte. Then

$$
-V_{\text {ohm }}=-E d_{\text {ele }}
$$

where $d_{\text {ele }}$ is the electrolyte thickness. Using the convention that $i$ is positive if flowing from anode to cathode, $\mathrm{E}$ is positive for field in the anode to cathode direction.

Let $i$ be the measured current density in the electrolyte and $i_{e}$ be the electronic current density in the electrolyte. The protonic current density $i_{p}$ is given by 


$$
i_{p}=i-i_{e}
$$

Under open-circuit condition, $i=0$, there are equal and opposite protonic and electronic current densities in the electrolyte, $i_{p}=-i_{e}$. This means that both protons and electrons flow through the electrolyte from the anode to the cathode side. Such binary flow cannot result from an electric field $E$ alone. There must also be proton and electron concentration gradients.

We assume that no ions other than protons have a concentration gradient. This means that for our assumed charge neutrality in the electrolyte, the proton and electron concentration gradients must be equal, so they can both be expressed by the same symbol $\frac{\partial n}{\partial x}$. We also assume that the change of concentration $d_{e l e}|\partial n / \partial x| \ll n_{e}$ or $n_{p}$, where $d_{e l e}$ is electrolyte thickness; otherwise the equations become nonlinear.

The proton and electron current densities are proportional to their individual gradients of chemical potential, not just gradients of electric potential. These current densities are given by

$$
\begin{gathered}
i_{p}=\sigma_{p} E-e D_{p} \frac{\partial n}{\partial x}=\sigma_{p} E-\mu_{p} k T \frac{\partial n}{\partial x} \\
i_{e}=\sigma_{e} E+e D_{e} \frac{\partial n}{\partial x}=\sigma_{e} E-\mu_{e} k T \frac{\partial n}{\partial x} \text { and } \\
i=i_{p}+i_{e}=\left(\sigma_{p}+\sigma_{e}\right) E-\left(\mu_{p}+\mu_{e}\right) k T \frac{\partial n}{\partial x}
\end{gathered}
$$

In these equations, $-e$ is the electron charge, the electron mobility $\mu_{e}$ is negative, $\mu_{p}$ is proton mobility, $\sigma_{p}$ is proton conductivity, $\sigma_{e}$ is electronic conductivity and the Nernst-Einstein relation

$$
q D=\mu k T
$$

was used in the equation where $q$ is carrier charge and $D$ is diffusion coefficient.

For short circuit, the electrons all go through the external circuit and so Eq. 41 becomes $i_{e s}=0$, giving

$$
k T\left(\frac{\partial n}{\partial x}\right)_{s}=\frac{\sigma_{e} E_{s}}{\mu_{e}}
$$

Plugging Eq. 44 into Eq. 40 gives

$$
i_{p s}=i_{s}=\left(\sigma_{p}-\frac{\sigma_{e} \mu_{p}}{\mu_{e}}\right) E_{s}
$$

Therefore, we expect that $i_{p}$ is proportional to $E$,

$$
i_{p}=\left(\sigma_{p}-\frac{\sigma_{e} \mu_{p}}{\mu_{e}}\right) E=\left(\sigma_{p}-\frac{\sigma_{e} \mu_{p}}{\mu_{e}}\right)\left(V_{0}-V_{a c t}-V_{c o n}-V\right) / d_{\text {ele }}
$$

We also know that

$$
i_{e}=-\sigma_{e} V / d_{\text {ele }}
$$

To find $V(i)$, we use Eqs. 46 and 47 and obtain

$$
\begin{aligned}
V(i)= & \left(V_{0}-V_{a c t}-V_{c o n}\right)-\frac{i d_{e l e}}{\sigma_{p}} \\
& -\left[\left(V_{0}-V_{a c t}-V_{c o n}\right)-\frac{i d_{e l e}}{\sigma_{p}}\left(1-\frac{\mu_{p}}{\mu_{e}}\right)\right] /\left(\frac{\sigma_{p}}{\sigma_{e}}+1-\frac{\mu_{p}}{\mu_{e}}\right)
\end{aligned}
$$

The mobility of electrons in the perovskite is much higher than that of protons, i.e. $\mu_{e} \gg \mu_{p}$, so that the $\mu_{p} / \mu_{e}$ term in Eq. 48 can be neglected. ${ }^{14,15}$ Combining Eqs. 34 and 48 gives

$$
\begin{aligned}
V(i)= & \frac{U}{q}-\left(\frac{k T}{q}\right) \ln \left[\frac{(b+i)(d+i)}{(a-i)(c-i)} e^{\left(U_{d} / 2 k T\right)}\right]-\frac{i d_{\text {ele }}}{\sigma_{p}} \\
& -\left[\frac{U}{q}-\left(\frac{k T}{q}\right) \ln \left[\frac{(b+i)(d+i)}{(a-i)(c-i)} e^{\left(U_{d} / 2 k T\right)}\right]-\frac{i d_{\text {ele }}}{\sigma_{p}}\right] /\left(1+\frac{\sigma_{p}}{\sigma_{e}}\right)
\end{aligned}
$$

The upper line of Eq. 49 is the voltage for zero electronic conductivity and the lower line gives the reduction in voltage due to electronic conductivity in the electrolyte.

\section{Comparison of Model Predictions With Experimental Results}

Now we put in some particular numbers and gas concentrations to calculate all the attempt current densities and $V(i)$ for a particular $\mathrm{H}$-SOFC. We keep in mind that $a, b, c$ and $d$ are equivalent current densities corresponding to attempt rate for forward and reverse reactions at the anode-electrolyte and cathode-electrolyte TPBs, respectively. We know that from Eq. 8

$$
a=\frac{1}{2} n_{H_{2}}\left(\frac{k T}{m_{H_{2}}}\right)^{1 / 2} f_{a} q\left(1-v_{\text {ele }}\right)\left[1-\left(a_{\text {ele }}^{3} n_{H, \text { ele }} / 3\right)-\left(a_{\text {ele }}^{6} n_{H, \text { ele }}^{2} / 9\right)\right]
$$

The $\mathrm{H}_{2}$ concentration at the plenum can be found by

$$
n_{H_{2}, p}=\left(\frac{p_{H_{2}} j_{1}-\frac{i S}{q}}{j_{\text {total }}}\right) n_{\text {total }, p}
$$

where $p_{\mathrm{H}_{2}}$ is the $\mathrm{H}_{2}$ inlet partial pressure, $j_{1}$ is the total inlet gas flow density, $j_{\text {total }}=j_{1}+j_{\mathrm{H}_{2} \mathrm{O}}$ is the total gas flow density when steam is picked up by flowing the gas through a water bubbler, $i$ is the cell current density, $S$ is the effective cathode area and $n_{\text {total, } p}$ is the total gas concentration in the plenum. The $\mathrm{H}_{2}$ concentrations at the anode-electrolyte interface of the cell can be found by solving the dusty-gas model which gives

$$
\begin{aligned}
n_{H_{2}}= & n_{H_{2}, p}-\frac{i w}{\phi q D_{K, H_{2}}} \tau^{3}-n_{N_{2}, p}\left(\exp \left(\frac{i w}{n_{1} \phi q D_{H_{2}, N_{2}, 1}} \tau^{3}\right)-1\right) \\
& -n_{H_{2} O, p}\left(\exp \left(\frac{i w}{n_{1} \phi q D_{H_{2}, H_{2} O, 1}} \tau^{3}\right)-1\right)
\end{aligned}
$$

where $n_{H_{2}, p}, n_{N_{2}, p}$ and $n_{H_{2} O, p}$ are the $\mathrm{H}_{2}, \mathrm{~N}_{2}$ and $\mathrm{H}_{2} \mathrm{O}$ concentration in the plenum, respectively, $w$ is the thickness of the supporting anode, $\phi$ is the anode porosity, $D_{K, H_{2}}$ is $\mathrm{H}_{2}$ Knudsen diffusion coefficient, $D_{\mathrm{H}_{2}, \mathrm{~N}_{2}, 1}$ is $\mathrm{H}_{2}$ and $\mathrm{N}_{2}$ binary diffusion coefficient under total pressure equals to 1 atm, $D_{\mathrm{H}_{2}, \mathrm{H}_{2} \mathrm{O}, 1}$ is $\mathrm{H}_{2}$ and $\mathrm{H}_{2} \mathrm{O}$ binary diffusion coefficient under total pressure equals to $1 \mathrm{~atm}$ and $\tau$ is the tortuosity. The detailed derivation of Eq. 51, Eq. 52 and tortuosity $\tau$ was published elsewhere. ${ }^{7}$ When the system is running under open-circuit voltage, the $\mathrm{H}_{2}$ concentration at the anode-electrolyte interface is the same as that in the plenum. The mass $m_{H_{2}}$ is $3.32 \times 10^{-27} \mathrm{~kg}$. The charge $q$ transferred per reaction of Eq. 4 is two electrons, i.e. $3.2 \times 10^{-19} \mathrm{C}$.

To find the fraction of oxygen sites at TPBs, $f_{a}$, we first find $d_{t}$, the TPB length per $\mathrm{cm}^{2}$ of electrolyte surface. The tortuosity of the supporting anode was $\tau=1.48$ which can be modeled as resulting from each pore being straight, but inclined at an angle $\cos ^{-1}(1 / \tau)$. The pore then meets the electrolyte at this angle and its intersection with the electrolyte is an ellipse of semimajor axis $\tau \bar{r}$ and semiminor axis $\bar{r}$. The pore area as it impinges on the electrolyte then is $\pi \tau \bar{r}^{2}$. The total pore number $n_{p}$ of pores impinging per $\mathrm{cm}^{2}$ of electrolyte is $n_{p}=\phi / \pi \tau \bar{r}^{2}=1.577 \times 10^{7} / \mathrm{cm}^{2}$. Each pore perimeter $P_{p}$ as it impinges on the electrolyte is, according to Marks' Handbook ${ }^{16}$ 
Table I. Protonic conductivity $\sigma_{p}$ and electronic conductivity $\sigma_{e}$, proton concentration $n_{H, e l e}$ and $O$ atom concentration on cathode $n_{O}$ used for the fitting in the model.

\begin{tabular}{|c|c|c|c|c|c|}
\hline$P_{H_{2}}$ & $20 \%$ & $40 \%$ & $60 \%$ & $80 \%$ & $100 \%$ \\
\hline$\sigma_{\mathrm{p}}(\mathrm{S} / \mathrm{cm})$ & $6.482 \times 10^{-3}$ & $7.639 \times 10^{-3}$ & $8.142 \times 10^{-3}$ & $8.395 \times 10^{-3}$ & $8.488 \times 10^{-3}$ \\
\hline$\sigma_{\mathrm{e}}(\mathrm{S} / \mathrm{cm})$ & $9.477 \times 10^{-4}$ & $1.004 \times 10^{-3}$ & $1.106 \times 10^{-3}$ & $1.223 \times 10^{-3}$ & $1.328 \times 10^{-3}$ \\
\hline $\mathrm{n}_{\mathrm{H}, \text { ele }}(\%)$ & 11 & 11.5 & 13 & 14.5 & 14.5 \\
\hline$P_{O_{2}}$ & $5 \%$ & $10 \%$ & $21 \%$ & $30 \%$ & $50 \%$ \\
\hline$n_{O}\left(\right.$ atom $\left./ \mathrm{m}^{2}\right)$ & $1.160 \times 10^{16}$ & $1.640 \times 10^{16}$ & $2.377 \times 10^{16}$ & $2.838 \times 10^{16}$ & $3.662 \times 10^{16}$ \\
\hline$\sigma_{\mathrm{p}}(\mathrm{S} / \mathrm{cm})$ & $5.233 \times 10^{-3}$ & $7.152 \times 10^{-3}$ & $8.591 \times 10^{-3}$ & $8.866 \times 10^{-3}$ & $9.609 \times 10^{-3}$ \\
\hline$\sigma_{\mathrm{e}}(\mathrm{S} / \mathrm{cm})$ & $1.058 \times 10^{-3}$ & $1.216 \times 10^{-3}$ & $1.369 \times 10^{-3}$ & $1.415 \times 10^{-3}$ & $1.598 \times 10^{-3}$ \\
\hline $\mathrm{n}_{\text {H,ele }}(\%)$ & 11.8 & 12.5 & 13.5 & 15 & 15 \\
\hline
\end{tabular}

$$
P_{p}=\pi \bar{r}(1+\tau)\left[1+\frac{1}{4} m^{2}+\frac{1}{64} m^{4}+\frac{1}{256} m^{6}+\cdots\right]
$$

where $m=(\tau-1) /(\tau+1)$, so $P_{p}=5.584 \times 10^{-4} \mathrm{~cm}$. Multiplying by $n_{p}$ yields $d_{t}=1.048 \times 10^{4} \mathrm{~cm}^{-1}$. If we take the $1 / 3$ power of $O^{2-}$ ion volume to be the spacing between the $O^{2-}$ ions, i.e. $\left(a_{\text {ele }}^{3} / 3\right)^{1 / 3}=a_{\text {ele }} / 3^{1 / 3}, 0.308 \mathrm{~nm}$ for $\mathrm{Ba}\left(\mathrm{Ce}_{0.8} \mathrm{Y}_{0.2}\right) \mathrm{O}_{3-\delta}$, then the fraction $f_{a}$ of electrolyte surface consisting of TPB is $3.231 \times 10^{-4}$.

For the proton concentration $n_{H, \text { ele }}$ in the electrolyte, we consider $n_{H, e l e}$ as an adjustable number in the model since $n_{H, e l e}$ should depend on temperature, gas concentrations at the electrolyte-electrode interface and current density $i . n_{H . e l e}$ also is expected to be under $20 \%$ of the $\mathrm{Ba}\left(\mathrm{Ce}_{0.8} \mathrm{Y}_{0.2}\right) \mathrm{O}_{3-\delta}$ molecular concentration, i.e. $a_{\text {ele }}^{3} n_{H, e l e}=0.20$, because the maximum uptake of protons should equal the $\mathrm{Y}^{+3}$ concentration in the $\mathrm{Ba}\left(\mathrm{Ce}_{0.8} \mathrm{Y}_{0.2}\right) \mathrm{O}_{3-\delta}$.

We will take $100 \% \mathrm{H}_{2}$ fuel gas inflow as an example and use $a_{\text {ele }}^{3} n_{H, e l e}=0.145$. Multiplying all the factors in Eq. 50, we have open-circuit forward attempt current density for Eq. 4

$$
a_{0}=59.77 \mathrm{~A} / \mathrm{cm}^{2}
$$

The reverse attempt current density is

$$
b_{0}=3^{2 / 3}\left(1-v_{\text {ele }}\right)^{2} f_{a} a_{\text {ele }}^{4} n_{H, \text { ele }}^{2} v q / 9=2.473 \times 10^{3} \mathrm{~A} / \mathrm{cm}^{2}
$$

The $\mathrm{O}$ atom concentration on the surface of cathode material can be found by Eq. 18. The results from different oxygen partial pressures are listed in Table I. The value of $U_{d}$ used for the calculation was obtained from the best fit of the model to our experimental data, which gives $0.1 \mathrm{eV}$.

Due to the thin cathode, not much information on concentration polarization effects on the cathode side can be obtained. To calculate forward attempt current density $c$ for the reaction at the electrolyte-cathode interface, we assume the fraction $f_{c}$ of $O^{2-}$ sites on the TPB is equal to $f_{a}$ and the porosity of the cathode is the same as for the anode, $44 \%$. The lattice constant of LSM is $0.392 \mathrm{~nm}$, so

$$
c_{0}=\frac{3^{2 / 3} n_{O} a_{c}^{2} a_{\text {ele }}^{4} n_{H, e l e}^{2}\left(1-v_{\text {ele }}\right) f_{c} q v}{18}=5.032 \mathrm{~A} / \mathrm{cm}^{2}
$$

The steam concentration at the cathode-electrolyte interface $n_{c, H_{2} O}$ is a function of operating current density because of the generation of exhaust gas from running the cell. We further assume steam concentration in the air that we used to run the cell is $2 \%, 66 \%$ relative humidity in the air, so that the fraction of steam in the air at the anodeelectrolyte interface is $0.02+i /\left(q n_{\text {total }}\right)$, where $n_{\text {total }}$ is the total gas concentration at the cathode-electrolyte interface. Therefore, we get

$$
\begin{aligned}
d_{0}= & \frac{1}{2} n_{c, H_{2} O}\left(\frac{k T}{m_{H_{2} O}}\right)^{1 / 2}\left[1-\left(\frac{a_{\text {ele }}^{3} n_{H, \text { ele }}}{3}\right)-\left(\frac{a_{\text {ele }}^{6} n_{H, \text { ele }}^{2}}{9}\right)\right] \\
& \times\left(1-v_{\text {ele }}\right)\left(1-\frac{n_{O} a_{c}^{2}}{2}\right) f_{c} q=0.406 \mathrm{~A} / \mathrm{cm}^{2}
\end{aligned}
$$

According to Eq. 25, we note that the current density $i$ is limited to a finite range, in which $-b \leq i \leq a$ (for anode limitation) and $-d \leq i \leq c$ (for cathode limitation) regardless of the value of $V$ as it runs from negative to positive infinity. Our calculation shows the output of the H-SOFC is limited by the attempt current density $c$, which refers to the reaction $2 \mathrm{H}^{+}+\mathrm{O}_{(S)}+2 e^{-} \rightarrow \mathrm{H}_{2} \mathrm{O}$, accordingly $5.032 \mathrm{~A} \mathrm{~cm}^{-2}$ for the maximum current density. For the SOEC mode, the system is limited by the attempt current density $d, 0.406 \mathrm{~A} \mathrm{~cm}^{-2}$. The number $d$ is relatively low due to the low steam concentration at the cathode-electrolyte interface. When the steam concentration increases the attempt current density $d$ will increase accordingly.

The enthalpy released by a hydrogen-oxygen reaction, $\mathrm{H}_{2}+\frac{1}{2} \mathrm{O}_{2} \rightarrow \mathrm{H}_{2} \mathrm{O}$, at $700^{\circ} \mathrm{C}$ can be found by using the best fit to the values for $800,900,1000,1100$ and $1200 \mathrm{~K}$ in Ref. 17 table A 5.4, using a quadratic polynomial trendline. The fitting gives the value $U_{700^{\circ} \mathrm{C}}=247,706 \mathrm{~J} \mathrm{~mol}^{-1}$. Therefore, the value $\frac{U}{q}$ in Eq. 49 can be calculated as

$$
\begin{aligned}
V_{0} & =\frac{U}{q}=247706 / N_{A} q=247706 /\left(6.023 \times 10^{23} \times 3.2 \times 10^{-19}\right) \\
& =1.285(\text { volt })
\end{aligned}
$$

For the numerical data of the model, we use Wolfram Mathematica 7.0 for the calculation. The protonic conductivities and electronic conductivities used in the model calculations were extracted from the $V(i)$ curves that we use to compare with our model. The results for changing $\mathrm{H}_{2}$ partial pressure while air was used at the cathode side, and changing $\mathrm{O}_{2}$ partial pressure while $100 \% \mathrm{H}_{2}$ was used at the anode side, at $700^{\circ} \mathrm{C}$ are listed on Table I. Details of the method used to calculate the proton conductivity and electron conductivity from the $V(i)$ curves will be published elsewhere. All the parameters that we use for this model are listed in Table II. Figures $3 a$ and $3 b$ compares the result from experiment and model for $\mathrm{H}_{2}$ and $\mathrm{O}_{2}$ partial pressure dependent tests at $700^{\circ} \mathrm{C}$. We see excellent agreement between the experimental and modeling data. The $n_{H, \text { ele }}$ used in the model for each $V(i)$ curve are listed in Table I. We notice that the calculated open-circuit voltages are about $0.05 \mathrm{~V}$ higher than those from the experiments. By looking at Eq. 50, when $i=0$, the only parameter that we can argue to lower the open-circuit voltage is to lower $a_{c}$ from $3.93 \AA$ down to $\sim 1 \AA$, not reducing the cathode material lattice constant but reducing the distance between $\mathrm{O}$ atoms on the cathode material surface. At the same time, we need to increase $U_{d}$ from 0.1 to $0.6 \mathrm{eV}$ to get a reasonable agreement with the experimental data. However, a lower $a_{c}$ seems to be consistent with an $\mathrm{O}$ atom that can sit randomly on the surface of the cathode material surface without the effect of Coulomb force from the ions of the cathode material. We have no further information of how $\mathrm{O}$ atoms interact with the surface of the cathode material. The disagreement of the open-circuit voltage can be treated as a defect of our model or it predicts the way that $\mathrm{O}$ atoms interact with the surface of the cathode material. This question, therefore, needs to be further investigated. 


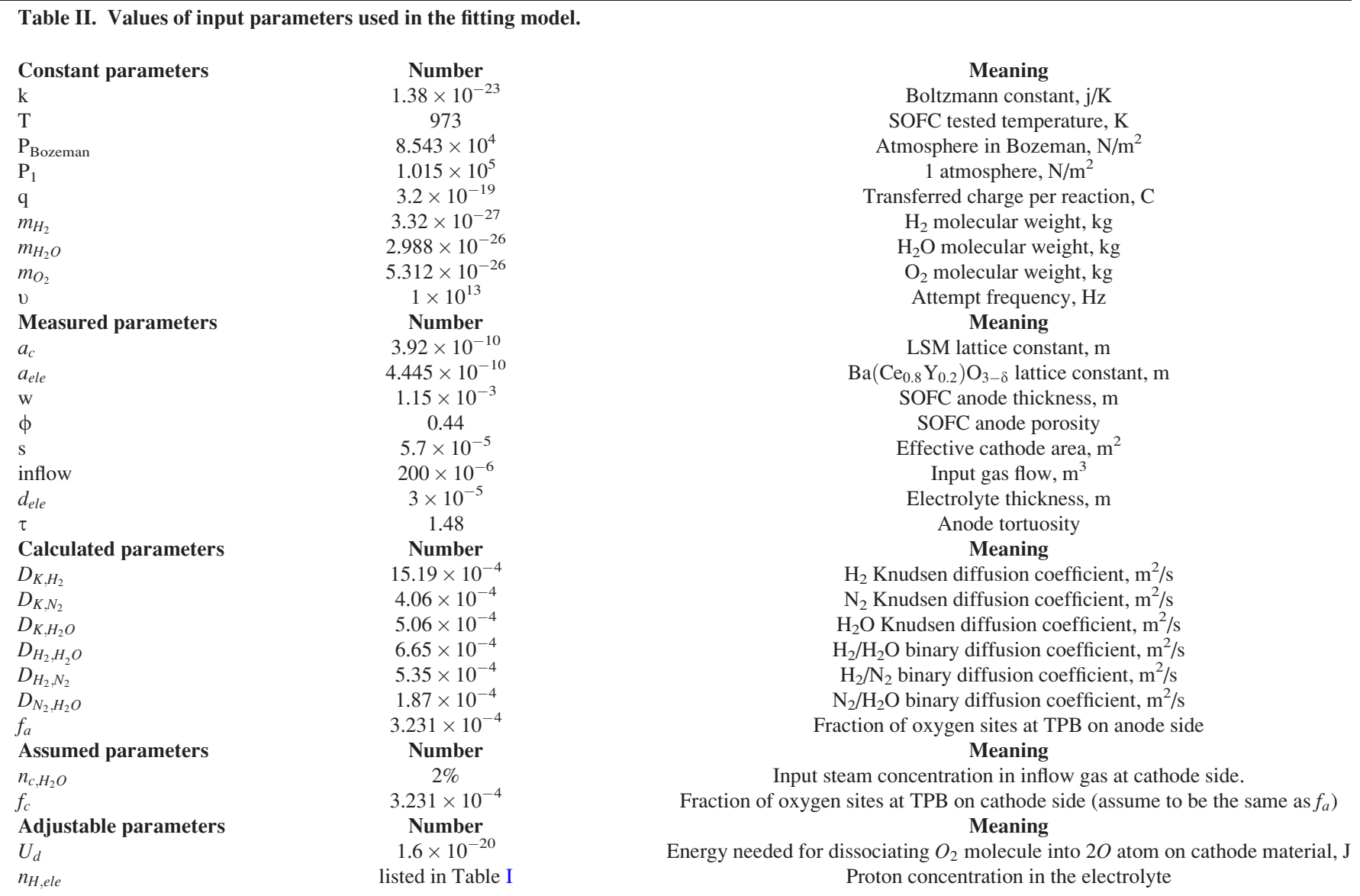

\section{Model Predictions for the Effects of Operating Parameters on H-SOFC Performance}

By using this validated model, the effects from different parameters can be studied. We first keep all the parameters the same as found by fitting to the experimental data and only change the electrolyte thickness. All the data presented here are using $100 \% \mathrm{H}_{2}$ as fuel gas and air as oxidative gas. Figure 5 shows the effect of changing the electrolyte thickness. As we can see, the H-SOFC curves are dominated by ohmic polarization when the electrolyte thickness is thicker than $20 \mu \mathrm{m}$. The effect of concentration polarization starts to come into play when the electrolyte thickness is thinner than $15 \mu \mathrm{m}$. The current density is finally limited to $5.032 \mathrm{~A} \mathrm{~cm}^{-2}$ by the attempt current density $c_{0}$ even when a thinner electrolyte is used. To increase the H-SOFC performance, Eq. 56 shows that the only two parameters that we can argue could be adjusted are $f_{c}$ and $n_{o}$. This means that the shortage of oxygen atoms at the cathode limits the current density. Therefore, to increase TPB length on the cathode side and oxygen atom concentration on the cathode material surface would be the methods to minimize this limitation and increase performance.

For the purpose of clearly seeing the polarization effects, we lower the electrolyte thickness from 30 to $15 \mu \mathrm{m}$ and also keep most other parameters the same as used for fitting to the experimental data. Figure 6 shows the effect of changing tortuosity. Since we assume the gas concentration on the cathode side is constant across the cathode due to its small thickness, the change of tortuosity in Fig. 6 only refers to the supporting anode. As we can see, the change of tortuosity does not affect the cell performance much until its value becomes higher than 2.5 . This is simply because there is only $\mathrm{H}_{2}$ inflow in the supporting anode of the H-SOFC, which results in the pressure gradient across the supporting anode by the effect of tortuosity being small. For O-SOFC, the production of steam on the anode side makes it suffer from concentration polarization even when the tortuosity of its supporting anode is low. The counterflow of fuel gas and steam makes the effect of tortuosity more obvious and requires more careful design for the supporting anode for O-SOFC.

The effect of changing the fraction of oxygen sites at TPB on the anode, $f_{a}$, and on the cathode, $f_{c}$, are shown in Figs. $7 \mathrm{a}$ and 7b. From the attempt current densities, we know that the performance of the whole cell is primarily limited by attempt current density $c$, i.e. limited by the reaction $2 \mathrm{H}^{+}+$ $O+2 e^{-} \rightarrow \mathrm{H}_{2} \mathrm{O}$. Figure 7a shows that the increasing or slight decreasing of $f_{a}$ does not change the performance of H-SOFC. However, if we further lower the $f_{a}$ down to 10 times smaller, the H-SOFC starts to encounter concentration polarization due to the lack of reaction sites for reducing hydrogen molecules into protons. On the other hand, Fig. $7 \mathrm{~b}$ shows that the increasing or decreasing of $f_{c}$ dramatically affects the performance of the $\mathrm{H}$-SOFC. It is easily seen that the cathode has much more sensitivity than the anode for the performance of H-SOFC. Therefore, the cathode of H-SOFC should be carefully engineered to increase its TPB to maximize the cell output.

The influence of the H-SOFC operating pressure is shown in Fig. 8a. The increasing power density comes from the increase of available $O$ atom concentration on the surface of the cathode material and the impinging rate of gas on the TPBs. The peak power density increases $10 \%$ when operating pressure doubles. Then, it increases 21.4 and $28.7 \%$ when operating pressure increases to 5 and $10 \mathrm{~atm}$, respectively. The effect from increasing operating pressure to get higher power output becomes lower when the pressure 


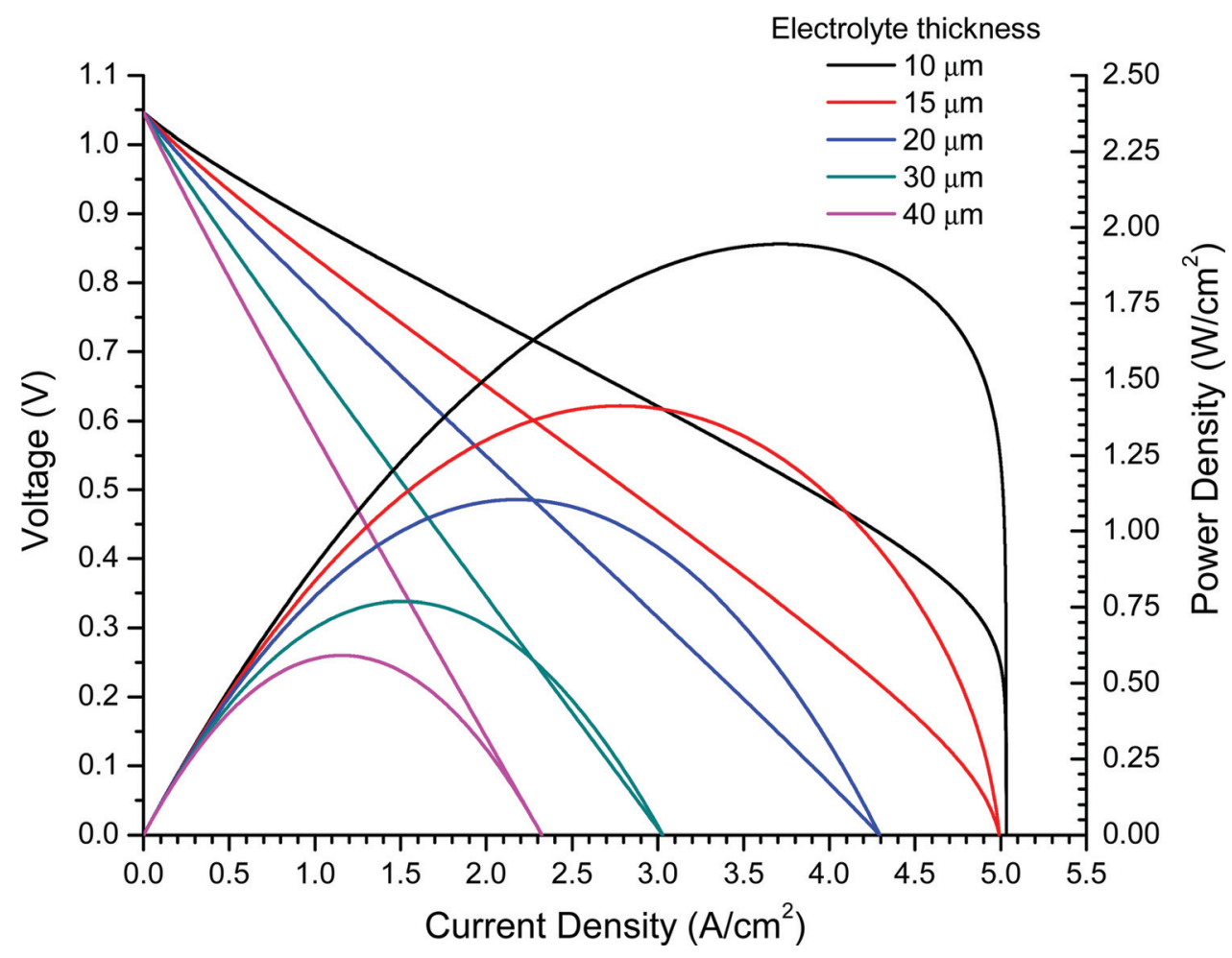

Figure 5. (Color online) The effect of changing electrolyte thickness of $\mathrm{H}$ SOFC.

gets higher. This result suggests that the benefit from increasing operating pressure might not always be positive when we consider the energy it takes to increase the operating pressure.

Figure $8 \mathrm{~b}$ shows the effect of steam concentration at the cathode-electrolyte interface for both SOFC and SOEC modes. For the SOFC mode, we can clearly see that the removal of steam at the cathode, by increasing flow rate of air, can increase the opencircuit voltage and power output. This is simply because the oxygen partial pressure at the TPB gets lower if the steam cannot be removed. In the SOEC mode, the cut-off current density is limited by attempt current density $d$, which means that even a large applied voltage could not drive the current density past this limitation. This is the current density corresponding to all steam molecules impinging on the TPB at the cathode-electrolyte interface ionizing into two $\mathrm{H}^{+}$and one $\mathrm{O}^{2-}$ successfully. From the structural point of view, H-SOEC should be a better electrolysis

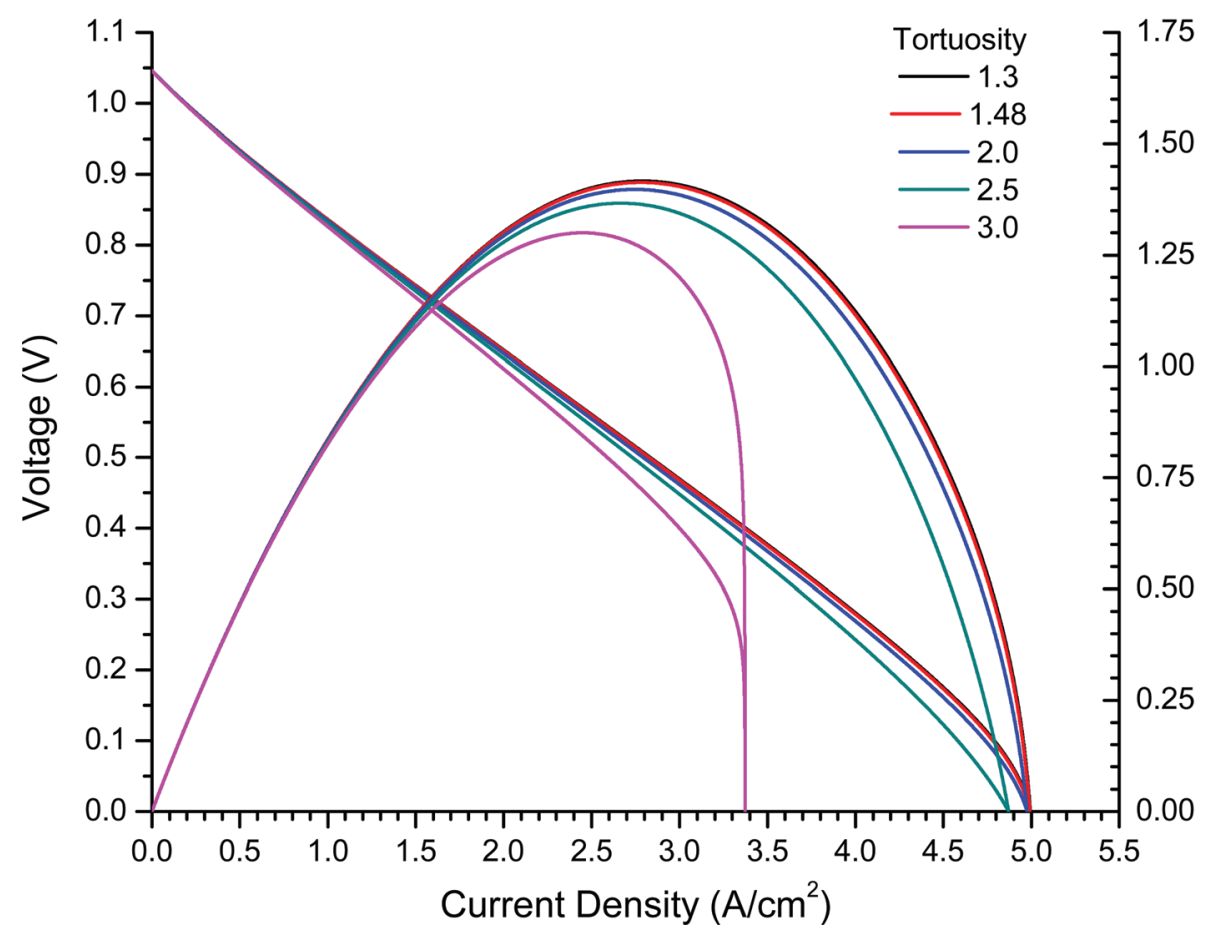

Figure 6. (Color online) The effect of changing tortuosity of $\mathrm{H}-\mathrm{SOFC}$ 

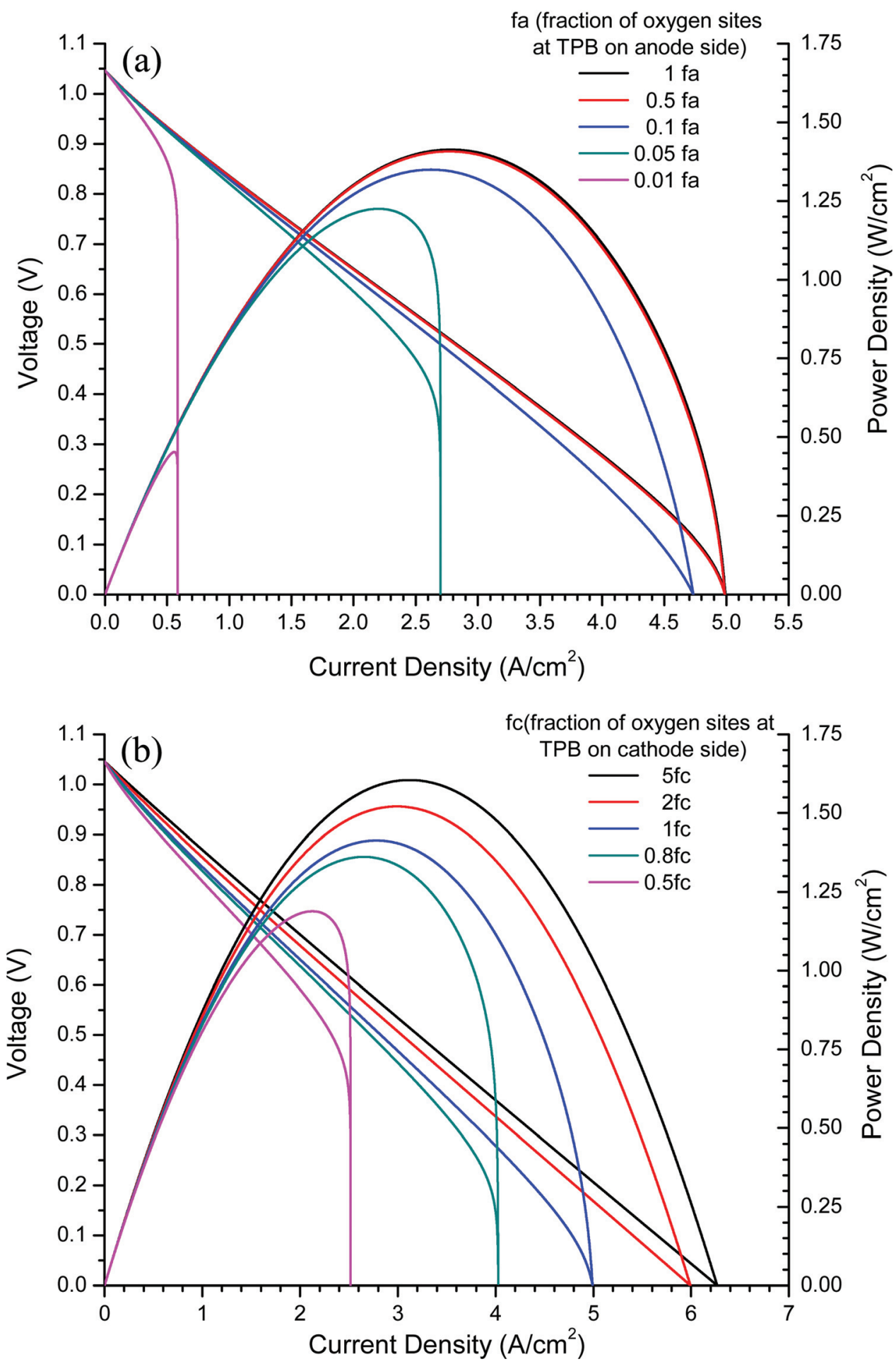

Figure 7. (Color online) The effects of changing fraction of oxygen sites at TPB on (a) anode side, $f_{a}$, and (b) cathode side, $f_{c}$. device than O-SOEC because the diffusion path for steam at the cathode side in H-SOEC is much shorter than at the anode side in O-SOEC.

Overall, our model suggests that the H-SOFC performance is affected more by the cathode than the anode. A cathode interlayer consisting of a mixture with similar volumes of cathode and electrolyte material can be used for increasing the TPB. However, proton conductive ceramics are famous for their difficulty to sinter; usually temperature above $1200^{\circ} \mathrm{C}$ is needed to trigger the sintering process and above $1400^{\circ} \mathrm{C}$ is needed to get highly dense ceramic when a sintering additive is used. ${ }^{18}$ On the other hand, cathode material is usually sintered at temperature $\sim 1000^{\circ} \mathrm{C}$ to keep its high porosity. Temperature higher than $1200^{\circ} \mathrm{C}$ can close up the pores in the cathode and lower the performance of the cell. If a cathode interlayer is simply applied and sintered at temperature $\sim 1000^{\circ} \mathrm{C}$, the contact between cathode interlayer and electrolyte might not be good enough for providing the conductive channels of protons, i.e. the ohmic polarization from the cathode-electrolyte interface will be 

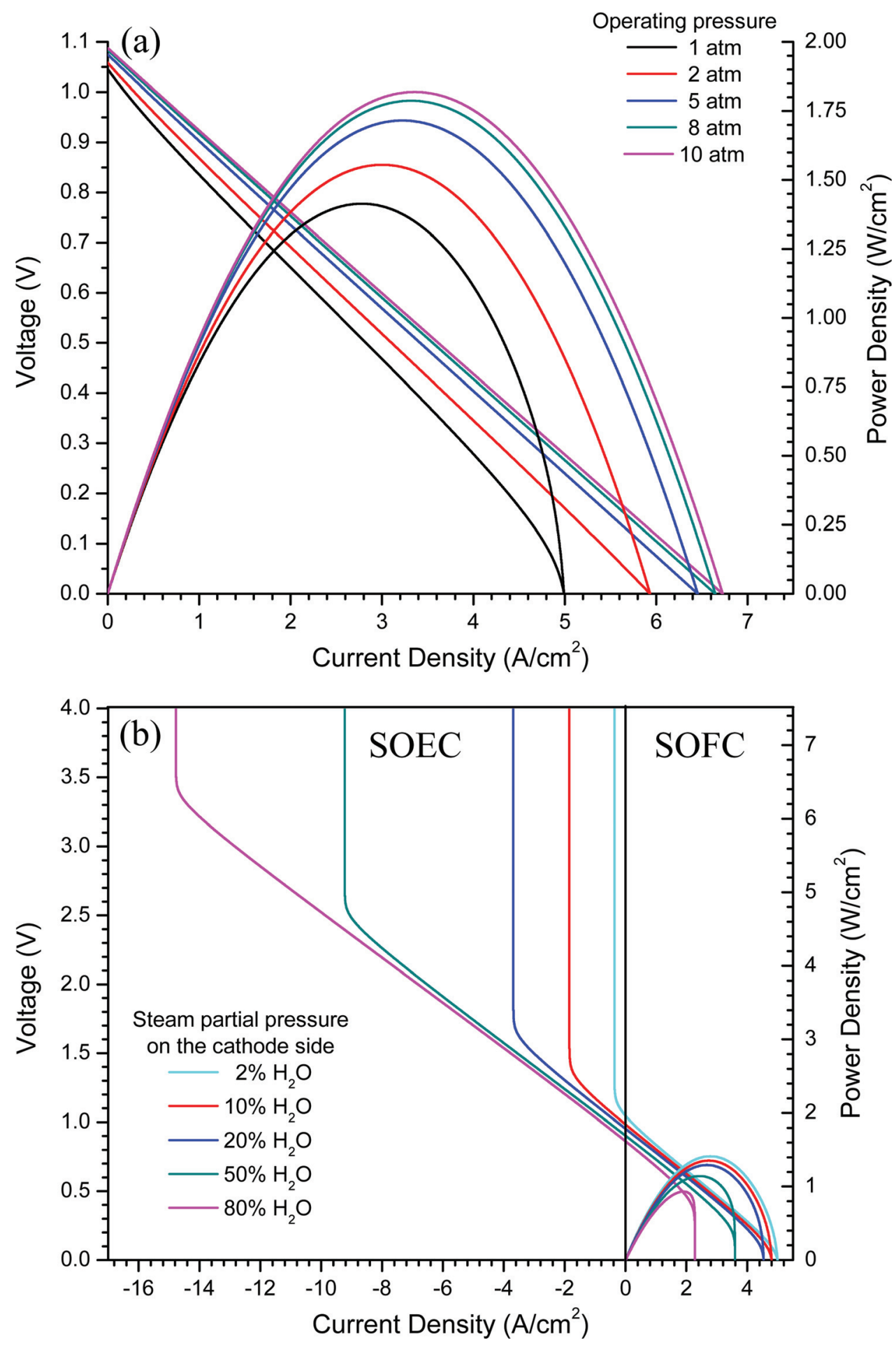

Figure 8. (Color online) The effects of changing (a) operating pressures and (b) steam concentration at cathode-electrolyte interface for both SOFC and SOEC modes. high for this situation. Some new idea for solving this problem should be developed and examined experimentally.

\section{Conclusions}

High performance $\mathrm{Ba}\left(\mathrm{Ce}_{0.8} \mathrm{Y}_{0.2}\right) \mathrm{O}_{3-\delta} \mathrm{H}$-SOFCs were made and tested under different temperatures and gas partial pressures. An electrochemical model was developed to study the $V(i)$ performance of the H-SOFC. The form that we derived for the $V(i)$ expression is different from the expressions that can be seen in the literature. ${ }^{8-13,19-21}$
Usually, the Nernst equation is used for deriving the contribution of concentration polarization where typically the partial pressure will appear. These partial pressure terms are related to the gas concentration terms in our expression for attempt current densities by the ideal gas law. The factors that are not related to gas concentrations are omitted in the usual literature expressions, but their effect shows up in a combined entropy and enthalpy term when proceeding to the logarithmic term. In our expression, $U / q$ is the total enthalpy release by the reaction $\mathrm{H}_{2}+\frac{1}{2} \mathrm{O}_{2} \rightarrow \mathrm{H}_{2} \mathrm{O}$. The released enthalpy is independent of cell construction and operating parameters, whereas the entropy 
depends on such parameters. By taking the factors unrelated to gas concentration into account, we include entropy in the prediction of $V(i)$. Therefore, the good agreement between the experimental data and model prediction provides evidence that our model is close to the $\mathrm{H}-\mathrm{SOFC}$ physical picture.

Parametric analyses were also performed to identify the key sources for improving the H-SOFC performance. The reducing of electrolyte thickness, i.e. reducing ohmic polarization, is found to be the major possible and easier way to improve the performance of the cell. Other than reducing electrolyte thickness, the performance of $\mathrm{H}-\mathrm{SOFC}$ is mostly restricted by the attempt current density $c$, which means the cathode of the H-SOFC controls the performance of the whole cell. The expression for attempt current density $c$ suggests that increasing the TPB length at the cathode-electrolyte interface and having more available $\mathrm{O}$ atoms on the cathode surface improve the performance of H-SOFC. Therefore, using a cathode interlayer and increasing $\mathrm{H}-\mathrm{SOFC}$ operating pressure are the ways to increase $\mathrm{H}$-SOFC performance.

\section{Acknowledgments}

The authors thank Dr. Stephen W. Sofie for the use of all his facilities. This work was supported by the United States Department of Energy, as a subcontract from Battelle Memorial Institute and Pacific Northwest National Laboratory under Award No. DE-AC0676RL01830.

\section{List of Symbols}

\begin{tabular}{|c|c|}
\hline$a$ & Anode forward attempt current density \\
\hline$a_{\text {ele }}$ & Lattice constant of electrolyte material \\
\hline$a_{c}$ & Lattice constant of cathode material \\
\hline$b$ & Anode reverse attempt current density \\
\hline$c$ & Cathode forward attempt current density \\
\hline$D$ & Diffusion coefficient \\
\hline$d$ & Cathode reverse attempt current density \\
\hline$d_{\text {ele }}$ & Electrolyte thickness \\
\hline$f_{a}$ & Fraction of oxygen sites at the anode-electrolyte TPB \\
\hline$f_{c}$ & Fraction of oxygen sites at the cathode-electrolyte TPB \\
\hline$i_{0}$ & Exchange current density \\
\hline$i_{0 a}$ & Anode-electrolyte TPB exchange current density \\
\hline$i_{0 c}$ & Cathode-electrolyte TPB exchange current density \\
\hline$i_{e}$ & Electronic current density in the electrolyte \\
\hline$i_{p}$ & Proton current in the electrolyte \\
\hline$k$ & Boltzmann's constant, $1.38 \times 10^{-23}$ \\
\hline$m_{i}$ & Molecule mass of the component $i$ \\
\hline$n_{\mathrm{H}_{2}}$ & $\mathrm{H}_{2}$ fuel gas concentration at the anode-electrolyte interface \\
\hline$n_{H, \text { ele }}$ & Proton concentration in the electrolyte \\
\hline$n_{c, \mathrm{H}_{2} \mathrm{O}}$ & The steam concentration on the cathode-electrolyte TPB \\
\hline$n_{\mathrm{O}_{2}}$ & Oxygen concentration in the cathode pore \\
\hline$n_{O}$ & $\mathrm{O}$ atom concentration on the cathode material surface \\
\hline$q$ & Charge transfer per reaction, $3.2 \times 10^{-19} \mathrm{C}$ \\
\hline$T$ & Absolute temperatuer $(\mathrm{K})$ \\
\hline$U_{a}$ & $\begin{array}{l}\text { Enthalpy released when the reaction } H_{2} \leftrightarrow 2 H^{+}+2 e^{-} \text {occurs } \\
\left(\mathrm{J} \mathrm{kg}^{-1}\right)\end{array}$ \\
\hline$U_{c}$ & $\begin{array}{l}\text { Enthalpy released when the reaction } 2 \mathrm{H}^{+}+\mathrm{O}+2 e^{-} \leftrightarrow \mathrm{H}_{2} \mathrm{O} \\
\text { occures }\left(\mathrm{J} \mathrm{kg}^{-1}\right)\end{array}$ \\
\hline$U_{d}$ & $\begin{array}{l}\text { Enthalpy released in the adsorption/dissociation combined reaction } \\
\left(\mathrm{J} \mathrm{kg}^{-1}\right)\end{array}$ \\
\hline
\end{tabular}

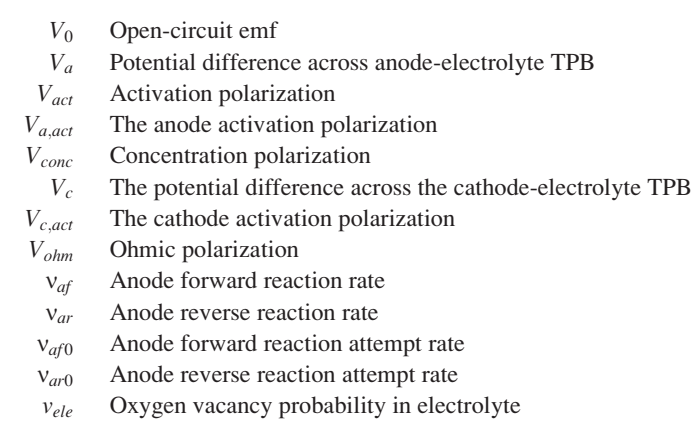

Greek letters

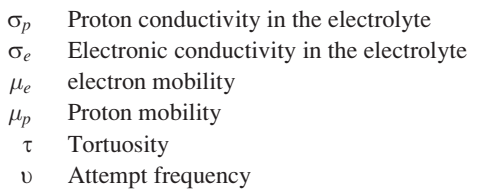

Abbreviations

SOFCs Solid oxide fuel cells

H-SOFCs Proton conductive solid oxide fuel cells

O-SOFCs Oxygen ion conductive solid oxide fuel cells

TPS Triple phase boundary

\section{References}

1. A. A. Ferreira, J. A. Labrincha, and J. R. Frade, Solid State Ionics, 77, 210 (1995).

2. Y.-P. Xiong, H. Kishimoto, K. Yamaji, M. Yoshinaga, T. Horita, M. E. Brito, and H. Yokokawa, Electrochem. Solid-State Lett., 13, B21 (2010).

3. S.-H. Park and H. I. Yoo, Phys. Chem. Chem. Phys., 11, 391 (2009).

4. M. Mogensen, N. M. Sammes, and G. A. Tompsett, Solid State Ionics, 129, 63 (2000).

5. A. Kruth and J. T. S. Irvine, Solid State Ionics, 162-163, 83 (2003).

6. A. Tomita, T. Hibino, and M. Sano, Electrochem. Solid-State Lett., 8, A333 (2005).

7. C.-L. Tsai and V. H. Schmidt, J. Power Sources, 196, 692 (2011).

8. M. K. H. Leung and D. Y. C. Leung, Fuel Cell 07, 4, 269 (2007).

9. M. Ni, M. K. H. Leung, and D. Y. C. Leung, J. Power Sources, 177, 369 (2008).

10. M. Ni, M. K. H. Leung, and D. Y. C. Leung, Int. J. Energy Res., 33, 943 (2009).

11. M. Ni, M. K. H. Leung, and D. Y. C. Leung, Energy Convers. Manage., 48, 1525 (2007).

12. H. Zhu, R. J. Kee, V. M. Janardhanan, O. Deutschmann, and D. G. Goodwin, J. Electrochem. Soc., 152, A2427 (2005).

13. W. G. Bessler, S. Gewies, C. Willich, G. Schiller, and K. A. Friedrich, Fuel Cells, 10, 411 (2010).

14. H.-I. Yoo, C.-R. Song, and D.-K. Lee, J. Eur. Ceram. Soc., 24, 1259 (2004).

15. K. D. Kreuer, Annu. Rev. Mater. Res., 33, 333 (2003).

16. L. S. Marks, Mechanical Engineers' Handbook, p. 107, McGraw-Hill, New York (1941).

17. X. Li, Principles of Fuel Cells, p. 545, Taylor \& Francis, New York (2006).

18. C.-L. Tsai, M. Kopczyk, R. J. Smith, and V. H. Schmidt, Solid State Ionics, 181 $1083(2010)$.

19. A. K. Demin, P. E. Tsiakaras, V. A. Sobyanin, and S. Y. Hramova, Solid State Ionics, 152-153, 555 (2002).

20. S. Assabumrungrat, W. Sangtongkitcharoen, N. Laosiripojana, A. Arpornwichanop, S. Charojrochkul, and P. Praserthdam, J. Power Sources, 148, 18 (2005).

21. A. Demin and P. Tsiakaras, Int. J. Hydrogen Energy, 26, 1103 (2001). 